\title{
1 Antiviral Functions of ARGONAUTE Proteins During 2 Turnip Crinkle Virus Infection Revealed by Image- 3 based Trait Analysis in Arabidopsis
}

4

\author{
Xingguo Zheng ${ }^{1}$, Noah Fahlgren ${ }^{1}$, Arash Abbasi ${ }^{1}$, Jeffrey C. Berry ${ }^{1}$, James C. \\ Carrington ${ }^{1 *}$ \\ ${ }^{1}$ Donald Danforth Plant Science Center, St. Louis, Missouri, 63132, United States of \\ America \\ *Author for correspondence: jcarrington@danforthcenter.org
}

\begin{abstract}
RNA-based silencing functions as an important antiviral immunity mechanism in plants. Plant viruses evolved to encode viral suppressors of RNA silencing (VSRs) that interfere with the function of key components in the silencing pathway. As effectors in the RNA silencing pathway, ARGONAUTE (AGO) proteins are targeted of by some VSRs, such as that encoded by Turnip crinkle virus (TCV). A VSR-deficient TCV mutant was used to identify AGO proteins with antiviral activities during infection. A quantitative phenotyping protocol using an image-based color trait analysis pipeline on the PlantCV platform, with temporal red, green and blue (RGB) imaging and a computational segmentation algorithm, was used to measure plant disease after TCV inoculation. This process captured and analyzed growth and leaf color of Arabidopsis plants in response to virus infection over time. By combining this quantitative phenotypic data with molecular assays to detect local and systemic virus accumulation, AGO2, AGO3, and AGO7 were shown to play antiviral roles during TCV infection. In leaves, AGO2 and AGO7 functioned as prominent non-additive, anti-TCV effectors, while AGO3 played a minor role. Other AGOs were required to protect inflorescence tissues against TCV. Overall, these results indicate that distinct AGO proteins have specialized, modular roles in antiviral defense across different tissues, and demonstrate the effectiveness of image-based phenotyping to quantify disease progression.
\end{abstract}

\section{Author Summary}

Plant viruses caused substantial losses in crop production and quality worldwide. Precisely measuring plant health is critical for better understanding the mechanisms underlying plant virus and host interactions. Advances in high-resolution imaging technologies and deep-learning tools have made acquiring and analyzing "big data" of 
disease traits possible. In this study, we have developed a high-throughput, imagebased trait phenotyping pipeline to quantify disease severity in Arabidopsis thaliana infected by Turnip Crinkle Virus (TCV). Our aim is to understand how the antiviral RNA silencing machinery is tuned to protect the host from invading virus infection. We focused on ARGONAUTE proteins, which are the effectors in the RNA silencing pathway. A mutant line of TCV with a dysfunctional silencing suppressor (P38) was used to investigate which ago mutation could compensate for the dysfunctional silencing suppressor and facilitate the development of disease symptoms. We demonstrated that specific AGO proteins contribute to protecting leaves from TCV infection in a non-additive manner. Our results also implied that distinct AGOs are required to function collectively to silence TCV in inflorescence tissues. More evidence is still needed to further understand how these antiviral AGOs interact with suppressor proteins molecularly during TCV infection.

\section{Introduction}

Plants can protect themselves against invasive virus infection through RNA silencing by targeting viral RNA for degradation [1]. This host silencing machinery is triggered by viral double-stranded RNAs (dsRNAs), which are cleaved by Dicer-like (DCL) nucleases associated with dsRNA binding proteins (DRBs) into 21-24 nucleotide RNA duplexes called viral small interfering RNAs (vsiRNAs). The vsiRNAs are then methylated and stabilized by HUA enhancer 1 (HEN1). One strand of these stabilized vsiRNAs is recruited into the RNA-induced silencing complex (RISC) containing ARGONAUTE (AGO) proteins, and then serves as the sequence-specific guide for specific AGOs to slice cognate viral RNAs [2, 3].

Most plant viruses have evolved to encode viral suppressors of RNA silencing (VSRs) that use varied mechanisms to target components in the silencing pathway [4]. One such mechanism is interference of AGOs that mediate antiviral silencing. Accumulating evidence indicates that various VSRs use diverse modes of action on AGO proteins, such as promoting AGO degradation [5-8], inhibiting the slicing activities of AGOs [9], or interfering with factors upstream of AGO activity such as RNA-dependent RNA Polymerase (RDR)-dependent silencing [10], obstructing siRNA-loaded RISC activity $[11,12]$, or indirectly repressing AGO protein level [13]. Since functional VSRs can mask host antiviral silencing effects, VSR-defective mutant viruses that can only successfully infect immunocompromised plants have been constructed to identify antiviral roles of key components in the silencing pathway during virus infection $[14,15]$. which have demonstrated antiviral roles [16]. AGO1 [14, 17-19] and AGO2 [15, 20-22] 
74 have been identified as prominent antiviral AGOs against several RNA viruses, while

75 other AGOs, including AGO5, AGO7, and AGO10, have limited antiviral roles in some

76 cases $[14,15]$. For DNA viruses, AGO4 is the main effector protein in the antiviral

77 silencing machinery [23, 24]. The full complement of AGOs with roles in antiviral

78 defense and how distinct antiviral AGOs are coordinated to silence different viruses in

79 different tissues remain to be fully determined.

Turnip crinkle virus (TCV) is a positive single-strand RNA virus belonging to the Carmovirus genus of the Tombusviridae family. The TCV genome encodes five proteins, including two replicase proteins (P28 and P88), two movement proteins (P8 and P9) and the coat protein (P38). The coat protein (CP) is multifunctional, as it has roles in virus movement [25, 26], serves as a virulence factor [27], and functions as a VSR to suppress antiviral silencing $[28,29]$. Other virus groups in the Tombusviridae encode separate VSR proteins, such as P19 from Tomato bushy stunt virus (TBSV) [19]. TCV systemically infects the susceptible Arabidopsis ecotype Columbia-O (Col-0), and causes disease symptoms that include severe chlorosis in leaves, stunted bolts and reduced biomass. A previous study reported that replacement of a single amino acid residue in the TCV CP P38 (R130T) disrupts its VSR function without affecting other functions [26]. The VSR-deficient TCV is unable to suppress the host antiviral silencing machinery, leading to a lack of disease symptoms in wild-type plants post-inoculation.

In this study, the roles of Arabidopsis AGO proteins in anti-TCV silencing were analyzed using genetic and image-based quantitative phenotyping approaches. Most previous pathological studies have relied on qualitative and subjective visual scoring systems to identify and assess disease phenotypes in plants [30]. A machine learning method [31] and other analysis tools in the open-source PlantCV platform [32, 33] were used to develop to detect subtle, reproducible differences in disease symptoms over time.

101

\section{Result}

Suppressor-deficient TCV is not able to elicit disease symptoms in wild-type host plants

Turnip Crinkle Virus (TCV) and other carmoviruses use their coat proteins (CPs) as VSRs to suppress host antiviral silencing [34, 35]. By replacing a single amino acid in TCV CP (P38) with its counterpart residue in TBSV CP (R130T) (Fig 1A), the VSR function of TCV CP (P38) is abolished [26]. Introducing this mutation (R130T) in the 
111 confirmed that this mutant virus (TCV CPB) lost its capacity to suppress host antiviral

112 silencing by quantifying and comparing the effects of TCV CPB in wild-type (Col-0) and

113 dc/2-1 dc/3-2 dc/4-2 (dc/2 dc/3 dc/4) triple mutant plants. In the $d c /$ triple mutant, three

114 DCL genes with roles in antiviral defense are dysfunctional, so it serves as a hyper-

115 susceptible control genotype. Parental TCV infection caused severe chlorosis in

116 Arabidopsis leaves in both wild-type control ( $\mathrm{Col}-\mathrm{O})$ and the $\mathrm{dc} /$ triple mutant plants,

117 while TCV CPB caused similar chlorosis symptoms only in the $d c /$ triple mutant (Fig 1B).

118

Turnip crinkle virus was from samples of inoculated rosette leaves and non-inoculated

120 cauline leaves using immunoblot assays with CP antisera (anti-P38). High levels of CP were detected in rosette leaves at 7 days post inoculation (dpi) and in cauline leaves at $14 \mathrm{dpi}$ of both Col-O and $d c / 2 d c / 3 d c / 4$ plants infected with parental TCV, even at 1:1000 dilution (Fig 1C). In contrast, low levels of CP were detected in rosette leaves of wild-type control plants inoculated with TCV CPB at lower dilutions (1:1 to 1:50) but not at higher dilutions (1:100 to 1:1000; Fig 1C). In non-inoculated cauline leaves of Col-0 plants, no observable CPB-P38 signal was detected at any dilution (Fig 1C). However, TCV CPB inoculation of $d c / 2 d c / 3 d c / 4$ mutant led to high levels of CP protein accumulation in the rosette and cauline leaves at each dilution ranging from 1:1 to 1:500 (Fig 1C). Notably, in $d c / 2 d c / 3 d c / 4$ mutants, no observable local or systemic CPB CP protein was detected when the TCV CPB inoculum was highly diluted (1:1000) (Fig 1C).

Parental TCV infection at any dilution had negative effects on the growth of both Col-0 and $d c / 2 d c / 3 d c / 4$ plants (Fig 1D). Morphologically, plants infected with TCV were shorter compared to non-infected plants (Fig 1D). In contrast, the TCV CPB mutant affected growth of only $d c / 2 d c / 3 d c / 4$ plants (Fig 1D). These results confirmed that the CPB (R130T) mutation in TCV CP attenuates VSR functions, and suggested that TCV CPB could be used in a genetic analysis to identify components of the silencing machinery necessary for antiviral defense.

\section{Image-based analysis of disease symptoms}

Before initiating a systematic screen of Arabidopsis ago mutants using TCV and TCV $\mathrm{CPB}$, a non-destructive, computer vision-based system using the PlantCV platform [33] was developed for high-resolution, quantitative assessment of disease symptom phenotypes over time (Fig 2A). The system was designed to identify individual plant leaves, measure their areas, lengths, and pixel color characteristics, and distinguish subtle differences in responses of plants with different genotypes over time. Top-down RGB images of individual plants were captured every other day, from one day preinoculation to seventeen days post-inoculation. The images were analyzed using a machine learning approach to segment plant from background and to classify plant 
pixels as "healthy" pixels (green color) or "unhealthy" chlorotic/necrotic pixels (any nongreen color; Fig 2B). The ratio of chlorotic/necrotic to total plant pixels was used to calculate the extent and severity of symptomatic rosette leaf tissue.

To validate the approach, wild-type (Col-0) and hyper-susceptible $d c / 2 d c / 3 d c / 4$ mutant plants were inoculated with parental TCV or TCV CPB mutant virus, or were mockinoculated (4 replicates per genotype and treatment combination). In the mockinoculated groups, the majority of rosette area remained healthy (green) over time in both $\mathrm{Col}-\mathrm{O}$ and $\mathrm{dcl} 2 \mathrm{dc/3} d \mathrm{~d} / 4$ plants (Figs 3A-B). Infection of both plant genotypes with parental TCV elicited local chlorosis at $7 \mathrm{dpi}$ and nearly complete chlorosis/necrosis by 17 dpi (Fig 3A-B). Discoloration caused by parental TCV infection appeared to be more severe in the $d c / 2 d c / 3 d c / 4$ mutant plants than in wild-type controls (Fig 3B). TCV CPB inoculation led to parental virus-like discoloration symptoms in the $d c /$ triple mutant plants (Fig 3B) but did not cause strong chlorosis symptoms in Col-0 plants (Fig 3A). Although an increased number of chlorotic pixels was observed in Col-0 rosettes at 15and 17-days post TCV CPB inoculation, the increase was visually less than that caused by parental TCV (Fig 3A).

Parental TCV infection led to reduced rosette area, based on total number of pixels (healthy plus chlorotic), indicating a decrease in biomass. Parental TCV significantly inhibited rosette growth over time in both wild-type and hyper-susceptible controls (Figs 3C-D) (Kolmogorov-Smirnov test, $p<0.05$ ). Similarly, TCV CPB led to a comparable decrease in rosette area in dc/2 dc/3 dc/4 mutants after $7 \mathrm{dpi}$, but not in Col-O (Figs 3BD). At $17 \mathrm{dpi}$, a significant difference in rosette area was detected between the two virus-treatments in Col-0, but not in $d c / 2 d c / 3 d c / 4$ plants (Fig 3E).

The percentage of unhealthy chlorotic/necrotic pixels in the whole rosette was also calculated. Both parental TCV and TCV CPB infection caused a significant increase in the percentage of unhealthy tissues over time in $d c / 2 d c / 3 d c / 4$ plants (Fig 3G), from approximately $0 \%$ at 5 dpi to nearly $100 \%$ at $17 \mathrm{dpi}$ (Fig 3G). In Col-0 plants, parental TCV infection also led to a gradual increase in chlorotic tissue, from approximately $0 \%$ at 5 dpi to nearly $60 \%$ at $17 \mathrm{dpi}$ (Fig 3F). In contrast, TCV CPB did not significantly change the percentage of chlorotic tissue in wild-type control plants over time (Fig 3F) (Kolmogorov-Smirnov test, $\mathrm{p}<0.05$ ). At $17 \mathrm{dpi}$, no significant difference in the percentage of unhealthy tissues was detected in TCV CPB-inoculated and mock treatment Col-0 plants (Fig $3 \mathrm{H})$.

Measurement of hue value of leaf color has been used to estimate chlorophyll loss caused by biotic and abiotic stress [36-38]. Hue value information extracted from the RGB images was used as a parameter to quantify chlorosis in rosette leaves. The hue 
value quantifies color in terms of angle around a circle, with values ranging from $0^{\circ}$ to $359^{\circ}$ [39], starting in the red color range. Yellow and green hue ranges span from $\sim 51^{\circ}$ to $80^{\circ}$ and $\sim 81^{\circ}$ to $140^{\circ}$, respectively. For each genotype and treatment combination, the mean proportion of pixels at each degree was plotted for each day. One primary peak centered around $100^{\circ}$, representing a green hue, was found in the histograms of mock-inoculated Col-O and $d c / 2 d c / 3 d c / 4$ plants over time (left panel, Figs $4 \mathrm{~A}-\mathrm{B}$ ). After $7 \mathrm{dpi}$, parental TCV infection caused a shift from a unimodal distribution of green hue values to a bimodal distribution that included a peak of yellow hue values in both genotypes (middle panel, Figs 4A-B). Notably, this green to yellow shift observed in $d c / 2$ $\mathrm{dcl} 3 \mathrm{dc} / 4$ mutants was more complete than that in Col-O plants at $17 \mathrm{dpi}$, suggesting the hyper-susceptible control leaves were more yellow than wild-type leaves (middle panel, Figs 4A-B). Similar to parental virus, TCV CPB induced a gradual shift from green to yellow in $d c / 2 d c / 3 d c / 4$ plants (right panel, Fig 4B). In contrast, this green-to-yellow shift was not observed in Col-0 plants inoculated with TCV CPB (right panel, Fig 4A).

The average hue value for whole plants in each treatment group was calculated. Parental TCV infection caused a temporal decrease in hue value in both $\mathrm{Col}-\mathrm{O}$ (from $100^{\circ}$ to $75^{\circ}$ ) and $d c / 2 d c / 3 d c / 4$ plants (from $100^{\circ}$ to $40^{\circ}$ ) (Figs 4C-D). TCV CPB induced a similar decrease in average hue value over time in $\mathrm{dc} / 2 \mathrm{dc} / 3 \mathrm{dc} / 4$ mutants, but did not significantly affect hue value in Col-O plants (Figs $4 \mathrm{C}-\mathrm{E}$ ). These results were consistent with the healthy/chlorotic classification-based results (Fig 3). These data indicate that the image-based growth and color trait measurement protocols were effective in quantifying virus-induced symptoms in Arabidopsis, and in distinguishing plant susceptibility or virus virulence phenotypes.

\section{Image-based analysis of TCV and TCV CPB infection in ago mutants}

As with the $d c / 2 d c / 3 d c / 4$ plants, we hypothesized that loss of ago genes with a function in anti-TCV silencing would be revealed by gain of susceptibility to the VSR-deficient TCV CPB mutant. A set of ten mutant plants with defects in each of the ten Arabidopsis AGO genes was inoculated with mock solution, parental TCV or TCV CPB, and imagebased traits and virus protein levels over an infection time-course were measured. Pairwise Kolmogorov-Smirnov (K-S) tests were done to compare time-series data.

Parental TCV infection caused a significant decrease in rosette size over time in all genotypes (Fig 5A). In contrast, TCV CPB inoculation led to three different effects, depending on genotype : a) no significant effect on rosette size overtime in ago4-2, ago5-2, ago8-1, ago9-5 and ago10-5; $b$ ) significant negative effects on rosette size change over time in ago2-1, ago3-2, zip-1 (ago7 mutant) and dc/2 dc/3 dcl4 control; or c) observable, but not significant, negative effects on rosette size change over time in 
ago1-27, ago6-3, and Col-0 plants (Fig 5A). After 7 dpi, a significant increase in the percentage of chlorotic/necrotic tissue was observed in all genotypes infected with parental TCV (Fig 5B), though the degree of increase varied in different genotypes (Fig $6 \mathrm{C})$. In the $d c / 2 d c / 3 d c / 4$ mutant, both parental and mutant TCV caused a comparable increase in the percentage of unhealthy tissue (Fig 5B). Among the ten ago mutants, TCV CPB significantly increased the percentage of unhealthy tissue only in ago2-1 and zip-1 mutants over time (Fig 5B). The ago1-27, ago3-2, ago4-2, ago5-2, ago6-3, ago81, ago9-5 and ago10-5 mutants were not responsive to TCV CPB inoculation (Fig 5B). Focusing on the data on $17 \mathrm{dpi}$, the effects caused by TCV CPB on rosette chlorosis in ago2-1 and zip-1 mutant could be directly displayed (Fig 6A) and statistically tested (Fig 6C) (Tukey post hoc test with $\alpha=0.05$ ). Similarly, by using this statistic test, we further confirmed that TCV CPB caused a significant decrease in ago2-1, ago3-2 and zip-1 rosette size at $17 \mathrm{dpi}$ (Fig 6B).

Another output of the phenotyping pipeline to evaluate leaf color is hue value (Fig 2A). The temporal changes in hue value in the ten inoculated ago mutants and control plants were measured over time (Fig 7). One primary peak at approximately $100^{\circ}$ (green) was observed in early stages for all genotype and inoculation combinations (-1 to $5 \mathrm{dpi}$ in each panel, Fig 7). In the mock-inoculated group, the primary peak remained at around $100^{\circ}$ in all genotypes throughout the time course. The green peak gradually shifted to yellow in each ago mutant and the two controls infected with parental TCV (7 to $17 \mathrm{dpi}$ in each panel, Fig 7). As expected, $d c / 2$ dc/3 dc/4 mutant plants infected with TCV CPB turned from green (peak at $100^{\circ}$ ) to yellow over time (blue area, dc/2/3/4 panel, Fig 7). Similarly, a complete green-to-yellow shift was measured in ago2-1 mutant plants infected with TCV CPB (blue area in ago2-1 panel, Fig 7). Gradual, partial shift from green to yellow was measured in zip-1 plants infected with TCV CPB (blue area in zip-1 panel, Fig 7). All other ago mutants (ago1-27, ago3-2, ago4-2, ago5-2, ago6-3, ago8-1, ago9-5 and ago10-5) inoculated with TCV CPB were similar to mock-inoculated plants (blue area, Fig 7).

To quantify the color shift observed above, average hue value was calculated. Pairwise Kolmogorov-Smirnov (K-S) tests were applied to compare time-series data. Parental TCV infection led to a gradual decrease in average hue value in all genotypes (Fig 8A), while TCV CPB caused a similar decrease only in ago2-1 and zip-1 mutants (Fig 8A) In addition, data at $17 \mathrm{dpi}$ further confirmed the negative effects of TCV CPB on average hue value in ago2-1 and zip-1 mutants (Fig 8B) (Tukey post hoc test with $\alpha=0.05$ ). 
To further investigate the roles of anti-TCV AGO proteins identified above in different tissues, immunoblotting assay was used to analyze viral coat protein (P38) levels in inoculated, non-inoculated (systemic) tissues, and inflorescence tissues of ago mutants and control plants. High levels of P38 were detected in leaves and inflorescence tissues of all ten single ago mutants and two control plants inoculated with parental TCV (left panel, Fig 9A; blue bars in Figs 9B-D). Local accumulation of CPB-P38 was only detected in ago1-27, ago2-1, and zip-1 mutant rosette leaves (right panel, Fig 9A, red bars in Fig 9B), at a comparable level with that in $d c / 2 d c / 3 d c / 4$ leaves (red bars in Fig 9B). In non-inoculated cauline leaves, systemic accumulation of CPB-P38 was only detected in ago2-1 and zip-1 mutants (Fig 9C), and at levels that were approximately half of that in $d c / 2 d c / 3 d c / 4$ mutants (Fig 9C). CPB-P38 in local and systemic leaves was also detected in two of four biological replicates of ago3-2 mutants (right panel, Fig 9A), though at a significantly lower level than that in ago2-1 mutants (Figs 9B-C). Notably, CPB-P38 was not detected in inflorescence tissues of any of the ten ago single mutant and Col-O plants (right panel, Figs 9A and 9C).

Next, to investigate if $\mathrm{AGO} 2$ and $\mathrm{AGO} 7$ had additive antiviral effects, and also to test if the antiviral activities of $A G O 1$, the previously reported antiviral AGO protein against TCV [14], was masked by AGO2 or AGO7, we performed immunoblotting experiments as described above in single, double or triple mutants combining ago1-27, ago2-1 and/or zip-1 mutant allele (Fig 10). First, parental TCV CPs accumulated at comparable levels in inoculated rosette, non-inoculated cauline leaves and inflorescence tissue in the single, double and triple ago mutants (Figs 10A-C). In local and systemic leaves, no significant differences in P38 level were observed between ago2-1, zip-1 and ago2-1 zip-1 double mutant (Figs 10B-C). These results suggested that AGO2 and AGO7 play non-additive antiviral roles in Arabidopsis leaves during TCV infection. To examine if AGO1, AGO2 or AGO7 play redundant antiviral roles, we checked if down-regulating ago1 had any enhancing effects on P38 accumulation in ago2 or ago7 mutants. We found no significant differences in local P38 level in rosette leaves between ago2-1 single, zip-1 single, ago1-27 ago2-1 double, ago1-27 zip-1 double and ago1-27, ago2-1 zip-1 triple mutant (Fig 10B). Similarly, combining the ago1-27 allele with an ago2-1 or zip-1 allele, or ago2-1 and zip-1, did not significantly affect the CPB-P38 accumulation level in systemic cauline leaves (Fig 10C). These results suggested that introducing the ago1-27 allele does not enhance or suppress the antiviral activities of AGO2 and AGO7 in Arabidopsis leaves. Similar to the observation in ago single mutants, no TCV CPB CPs were detected in the inflorescence clusters of the double and triple mutants tested (Fig 10D). 
The effects of TCV infection on plant height in ten ago mutants, the $d c /$ triple mutant and wild-type plants inoculated with parental and VSR-deficient TCV were measured. Growth curves were plotted from one day pre-inoculation to $21 \mathrm{dpi}$ as described previously [40]. Pairwise Kolmogorov-Smirnov (K-S) tests were done to compare timeseries data.

\section{Under mock treatment, plant height was greater than $30 \mathrm{~cm}$ in most genotypes at 21} $\mathrm{dpi}$, except in ago1-27 and $d c / 2 d c / 3 d c / 4$ mutants (Figs 11A and 11C). TCV infection led to reduced plant height $(<10 \mathrm{~cm})$ starting around $7 \mathrm{dpi}$ in all genotypes (Fig $11 \mathrm{~A})$. The TCV CPB mutant virus had only minor effects on height of inoculated Col-O plants over time, but strongly affected height in $d c / 2 d c / 3 d c / 4$ mutant plants (Figs $11 \mathrm{~A}-\mathrm{C}$ ). Mild inhibition of height caused by TCV CPB was observed at intermediate time points (Tukey post hoc test, $\alpha=0.05$, Fig 11B), though no effects on plant height were detected at $21 \mathrm{dpi}$ (Fig 11C).

The TCV CPB mutant also strongly inhibited plant height in ago2-1 and zip-1 mutants (Fig 11A). In ago2-1 mutants, TCV CPB infection had parental virus-like effects on plant height at 13 and $21 \mathrm{dpi}$ (Figs $11 \mathrm{~B}-\mathrm{C}$ ). In zip-1 mutants, the suppressor-deficient virus led to an approximately $60 \%$ decrease in plant height compared with mock-inoculated control plants at both time points (Figs 11B-C). In ago3-2 mutant plants, TCV CPB caused modest reduction of reduced height at both $13 \mathrm{dpi}$ and $21 \mathrm{dpi}$ (by Tukey post hoc test with $\alpha=0.05$, Fig 11C). TCV CPB had little or no effects on plant height in ago127, ago4-2, ago5-2, ago6-3, ago8-1, ago9-5, or ago10-5 mutants.

\section{Discussion}

Precisely measuring biotic and abiotic stress traits is important to understand the impact of the environment, pathogens and pests on plant growth and development, and to assist breeders to improve crops in modern agriculture. Objective, reproducible, and accurate quantification of disease severity is important for development and testing of disease-resistant crops. Various methods to estimate disease severity in plants are in use [30, 41-43], though many of these are dependent on qualitative or subjective observations, or require destructive sampling of plant material. Recently, some nondestructive, image-based analysis methods have been developed to measure plant morphology [44-46]. Machine learning techniques have been applied to efficiently process color trait data from large numbers of images, and to link the inputs to the outputs mathematically $[47,48]$. Here, we integrated a machine learning algorithm and analysis tools in the open-source phenotyping platform PlantCV $[32,33]$ to develop an 
image-based disease trait analysis pipeline to objectively measure disease severity in Arabidopsis plants infected by viruses.

In this pipeline, the naïve Bayes machine learning method [31, 32] was used for segmenting plant tissue from background in an image at pixel-level. First, four naïve Bayes classifiers were defined: 1) green plant pixels ("healthy"); 2) chlorotic/necrotic plant pixels ("unhealthy"); 3 ) blue mesh background pixels; 4) other background pixels. Since the light intensity condition was relatively constant, the color information of pixels from small number of sample images was sufficient for generating the training dataset to cover the range of variation of all images. In the training session, four classes of pixels could be segmented simultaneously. In addition, the naïve Bayes segmentation process is robust across large number of images due to its probabilistic nature. This pipeline is also simple and computationally expedient. The output of the pipeline could be statistically analyzed to quantify plant size, the proportion of unhealthy tissue, and leaf color. This non-destructive phenotyping pipeline enables visualization and quantification of disease symptom development over time from large numbers of plants.

In this study, the image-based disease analysis method was put to the test in wildtype and mutant Arabidopsis plants, with defects in AGO genes, infected by parental and VSR-defective TCV. Detailed phenotyping of inoculated Arabidopsis ago mutant plants was done. Ten AGO genes are encoded in the Arabidopsis genome and they are grouped into three major clades: AGO1/5/10, AGO2/3/7, and AGO4/6/8/9 [3]. Combining disease phenotyping and biochemical results, AGO2 and AGO7 were identified as prominent factors during anti-TCV defense, along with minor antiviral roles of AGO3. The results with AGO2 and AGO7 are consistent with previous studies [14, 20]. Using the VSR-defective virus, the antiviral role of $A G O 7$ was relatively minor compared to that of AGO2. AGO2 and AGO7 were non-additive in leaves (Fig. 10). However, movement of the TCV mutant to inflorescence tissues was still inhibited in ago2 ago7 double mutant, but not in dc/2 dc/3 dc/4 mutants (Fig. 10). These results implied that other AGOs not tested in the current genetic combinations are involved to restrict TCV movement to, or accumulation in, inflorescence tissues.

\section{AGO1 was previously considered as a prominent antiviral factor against several viruses} $[14,17,18]$. However, we observed that the ago1-27 mutant was not susceptible to the VSR-defective TCV, indicating that full AGO1 activity is clearly not necessary for antiTCV function. Since AGO1 is critical for regulating gene expression in numerous developmental and physiological pathways [3], ago1 null alleles are lethal and difficult to test. Thus, in the hypomorphic ago1-27 mutant, either enough functional AGO1 protein is present to contribute to suppression of the mutant TCV, or there is little or no role for AGO1 in the anti-TCV response. It should be noted that disruption of full AGO1 function 
can up-regulate of AGO2 expression [17, 20], meaning that AGO2 levels in the ago1-27 mutant could affect antiviral function of AGO2. Therefore, our data do not exclude possible direct or indirect roles for AGO1 during TCV infection.

Our finding that ago2 mutants were more susceptible to the VSR-defective TCV provided additional evidence that $\mathrm{AGO} 2$ functions as an important antiviral effector against a broad spectrum of plant viruses [15, 20-22]. We also found minor effects of AGO3. A recent report proposed that AGO3 was not involved in the antiviral pathway against cucumber mosaic virus (CMV) [49], though this conclusion is complicated by the use of parental CMV containing a functional VSR, which could possibly mask the effects of AGO3. AGO3 and AGO2 belongs to the same phylogenetic clade, and these two genes are closely linked in the Arabidopsis genome. AGO3 has slicer activity against viral RNAs in vitro [50] and binds siRNAs derived from Potato spindle tuber viroid in vivo [51]. Therefore, some degree of antiviral activity of AGO3 against TCV, as revealed using the VSR-defective virus, might not be surprising. However, to further investigation with combinations of mutations, such as with an ago2 ago3 double mutant, would be informative.

AGO7 binds miR390, which targets TAS3 transcripts for biogenesis of tasiRNAs [5255]. AGO7 has also been shown to affect antiviral function $[14,15]$, though its role is not clear. AGO7 has slicer activity [56], but it is not clear if AGO7 directly interacts with viral siRNAs or genomic (or subgenomic) RNAs during infection.

Our findings partly agreed with a previously reported modular, tissue-specific mode of antiviral AGO function against Turnip mosaic virus (TuMV), with AGO2 possessing a major role and AGO10, AGO5 and AGO7 having minor effects [15]. With TCV, both AGO2 and AGO7 are influential in protecting leaves, along with minor contributions from AGO3. This may reflect the fact that the VSRs encoded by different viruses use different molecular strategies to affect antiviral effector functions [57]. HC-Pro from TuMV was found to sequester viral siRNAs away from multiple AGO proteins [15]. Previous reports suggested that TCV infection led to expression changes of many endogenous genes, including AGO genes [58]. AGO2 expression was induced by TCV infection [20]. In contrast, TuMV did not affect AGO accumulation [15]. Therefore, the availability and coordination among antiviral AGOs against different viruses could possibly be virusdependent.

Furthermore, the capacity to physically interact with AGO protein was considered to be critical for the VSR functions of TCV CPs (P38) [59]. However, the VSR function of P37, coat protein of Pelargonium line pattern virus, was shown to be dependent on its SRNA binding capacity instead of its physical interactions with AGO protein [60]. Here, we 
423 showed that multiple AGOs participate in anti-TCV silencing. More molecular evidence

424 is needed to understand how P38 interacts with these AGOs to suppress antiviral

425 silencing machinery.

426

427 Regardless of precise antiviral mechanisms in play against TCV, the image-based

428 phenotyping system developed here was shown to be useful in delineating both major

429 and minor contributions of AGOs during virus infection over time. This should

430 encourage development and refinement of additional tools and readouts to measure

431 more effects and responses, even before visible symptoms of disease are detectable.

432 Closing the gap between knowledge at the genetic and molecular levels with phenotypic

433 effects during pathogen infection and non-pathogen colonization should yield

434 considerable new insights into host-microbe interactions.

\section{Conclusion}

Precise phenotyping methods to measure biotic and abiotic effects on plant health are important to develop. A high-throughput, image-based disease trait phenotyping pipeline to quantify virus-induced symptoms in Arabidopsis was developed. Combined with infectivity experiments using both parental and VSR-defective TCV variants, AGO2 and AGO7 were identified as the most prominent antiviral AGO proteins against TCV, while AGO3 was found to have a minor effect on antiviral silencing. These and previous data support the idea that multiple AGOs are recruited and programmed during antiviral defense in unique ways against different virus species.

\section{Plant materials}

All Arabidopsis plants used in this study (including all mutant lines) were in the Columbia-O (Col-O) background and were grown in growth chambers under long day (16-hour light/8-hour dark) conditions, at $22^{\circ} \mathrm{C}$ and $75 \%$ relative humidity. The ten ago mutants used in this study were described previously: ago1-27, ago2-1, ago3-2, ago4-2, ago5-2, ago6-3, zip-1, ago8-1, ago9-5 and ago10-5 [15]. Double and triple ago mutants were generated by crossing between single ago mutants. The $d c / 2-1 d c / 3-1 d c / 4-2$ triple 
457 Nicotiana benthamiana plants were grown in growth chambers under long day (16-hour 458 light/8-hour dark) conditions, at $22^{\circ} \mathrm{C}$ and $75 \%$ relative humidity.

\section{DNA plasmids}

The pSW-TCV plasmid is equivalent to pPZP212-TCV described before [26] (pSW is derived from pPZP212 plasmid by deleting most of RE sites with its MCS). The pSWTCV CPB construct incorporates the mutation (R130T) in pSW-TCV CP region [26]. TCV CP fragment was amplified from pSW-TCV and pSW-TCV CPB using one set of primers: P38_L (ATGGAAAATGATCCTAGAGTCCG) and P38_R (CTAAATTCTGAGTGCTTGCCATTT). P38 PCR segments were sent to GENEWIZ to confirm the CPB mutation by using sequencing primer P38_L (ATGGAAAATGATCCTAGAGTCCG).

Virus infection assays

Plasmids carrying TCV DNA clones were transformed into Agrobacterium tumefaciens (GV3101) and then infiltration was done on $N$. benthamiana leaves for viral inoculum preparation. The infected $N$. benthamiana leaves were grinded in $200 \mathrm{mM} \mathrm{NaOAc}$ solution ( $\mathrm{pH} 5.2$ ) at $4^{\circ} \mathrm{C}$. The grinded mixtures were centrifuged at $4^{\circ} \mathrm{C}$ to collect the supernatant. Next, 40\% PEG-8000 (Polyethylene glycol; MW:8000) in 1M NaCl solution was added to the supernatant and incubated on ice overnight. Virion pellet were collected by centrifuging and resuspended in 10mM NaOAc ( $\mathrm{pH} 5.5)$ solution for inoculum stock. The inoculum stocks were aliquoted into equal volumes and stored at $80^{\circ} \mathrm{C}$. The same set of inoculums (1:10 dilution with $10 \mathrm{mM} \mathrm{NaOAc}$ pH5.5) were used to inoculate all genotypes in this study. The $A$. thaliana plants to be inoculated were approximately two weeks old. The ago1-27 mutant plant was planted one week earlier than other genotypes. The four largest rosette leaves were inoculated by gently rubbing carborundum dusted on the leaf surface. Plants under different treatments were placed on separate benches to avoid possible cross contaminations in the growth chamber.

\section{Protein blot assays}

To measure local CP accumulation, four inoculated rosette leaves per plant were collected at 7 days post inoculation (dpi) and pooled into a single sample. For systemic $\mathrm{CP}$ accumulation, the four largest non-inoculated cauline leaves or five to six inflorescence clusters per plant were collected at $14 \mathrm{dpi}$ and pooled into a single sample. Four biological replicates were randomly collected from each genotypetreatment group. Total protein was extracted and normalized to $0.5 \mu \mathrm{g} / \mu \mathrm{l}$. Protein 


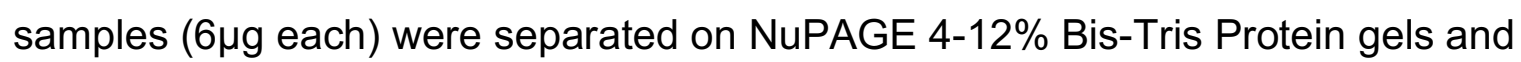
subsequently transferred to nitrocellulose blotting members $(0.45 \mu \mathrm{m}$, GE Healthcare Life Science) for protein detection with corresponding antibodies. TCV CP was detected using anti-TCV-P38 serum (F. Qu, personal communication) at a dilution of 1:20,000 and the large subunit of rubisco protein was detected using anti-rubisco (plant) antibody in chicken (Sigma-Aldrich) at 1:10,000. The blots were incubated with goat anti-rabbit IgG-HRP conjugate (GE Healthcare) secondary serum at a dilution of 1:10,000 to detect TCV CP and with rabbit anti-chicken $\lg \mathrm{Y}(\mathrm{H}+\mathrm{L}) \mathrm{HRP}$ conjugate secondary antibody (Thermo Fisher) at a dilution of 1: 10,000 to detect rubisco protein. TCV CP and rubisco protein were detected on the same blot. Quantification of western blot images was done by normalizing all other bands relative to $d c / 2-1 d c / 3-1 d c / 4-2$ (P38 band/ loading control band) using ImageJ software [60].

\section{Manual plant height measurement}

Measurement of plant height was done manually using a cubic ruler to measure from the rosette plane to the top of the main plant stem [62]. Data were collected every other day from the day of virus inoculation to $21 \mathrm{dpi}$.

\section{RGB Image acquisition}

The soil in the growth pots was covered by a blue mesh (commercially available from Amazon.com), leaving a hole in the center for the plant to grow out (Fig 2B). Meshed plants were bottom-watered to avoid water splash on plant tissues. Images of plant rosettes were manually acquired using a Canon Digital Rebel XT DSLR camera with EF-S 18-55mm f2.5-5.6 lens (0.60 s exposure, F/14, ISO100) on a Kaiser RS1 Copy Stand. The flash function was kept off and fixed ambient light was used in a closed room to minimize illumination discrepancies between images. An X-rite ColorChecker Digital SG was placed next to the plant as a color reference and correction. Images were stored in the native RAW format and also high-resolution JPG format. Plants were removed from the growth chamber $(10 \mathrm{am}-11 \mathrm{am})$ and any bolts above the rosette plane were removed before imaging. Stationary plant rosettes were imaged every other day from one day before inoculation to $17 \mathrm{dpi}$.

\section{Image Analysis}

Color images of individual mock- or virus-infected Arabidopsis plants were processed using the Plant Computer Vision (PlantCV) package to quantify the progression of disease symptoms [32, 33]. PlantCV v2.1 (commit: 
d553a2c1c6bd29e734d19898e3e9ac4fcff40aa9) was used. Image analysis was done in parallel using HTCondor v8.6.8 [63].

The PlantCV naive Bayes machine learning method [31, 32] was used to segment image pixels into four classes: 1) green plant pixels ("healthy"); 2) chlorotic/necrotic plant pixels ("unhealthy"); 3) background pixels from the blue mesh material used to cover the soil, and 4) all other background pixels. The PlantCV naive Bayes classifier was trained using pixel red, green, blue (RGB) color values from 1980 and 3779 pixels manually sampled from the background and foreground classes, respectively, from multiple images using the ImageJ pixel picking tool [64] as described in [32]. The training data was used to calculate probability density functions (PDFs) using kernel density estimation (KDE) for each class in the hue, saturation, and value (HSV) color space [32]. During image analysis, the PDFs were used to parameterize the naive Bayes classifier to segment images into the four output classes (one binary image per class).

After segmentation, the blue mesh was used to automatically identify the position of the pot within each image. Morphological erosion was used to reduce noise in the classified blue mesh pixels. A universal region of interest was used to keep blue mesh connected components in the center area of each image since the pot was consistently centered in each image. The remaining blue mesh connected components were flattened into a single object and were used to create a pot binary mask using the bounding rectangle area of the blue mesh. Padding was added to the minimum bounding rectangle area using morphological dilation. The resulting pot binary mask was used to mask the "healthy" and "unhealthy" binary masks to remove misclassified background pixels. The "healthy" and "unhealthy" binary images were further filtered to remove background pixels misclassified as foreground pixels using a size-based filter that removed small connected components (300 and 1000 pixels for "healthy" and "unhealthy" binary images, respectively). A universal region of interest was used to keep connected components in the center area of each image since the Arabidopsis plants were consistently centered in each image. The resulting cleaned binary image for the "healthy" and "unhealthy" classes were used to measure the area of green and chlorotic/necrotic pixels in each image.

Additionally, the union of the "healthy" and "unhealthy" plant pixels was calculated to create a combined plant mask. The input RGB images were converted to HSV color space and the frequency distribution of hue color values for each plant at each timepoint was extracted from the pixels that overlapped the combined plant mask. The hue color histograms were plotted in R v3.4.4 using the libraries ggplot2, ggridges, reshape2, and 
568 dplyr [65-69]. The PlantCV and R analysis code and result files are available at GitHub

569 (https://github.com/carringtonlab/tcv-image-analysis).

\section{Statistics}

571

572 All statistical analysis was performed in R v3.4.4.

\section{Acknowledgments}

We are grateful for Dr. Feng Qu (Department of Plant Pathology, The Ohio State

576 University, USA) for providing pSW-TCV plasmids and anti-P38 antibody. We thank

577 Kerrigan B. Gilbert, Dr. Dan Lin, Dr. Steen Hoyer and Dr. Kira Veley for support and

578 critique of the study. We also thank Robyn Allscheid for constructive editorial advices on

579 this manuscript.

The authors have declared no conflicts of interests.

This study was supported by funding: National Institutes of Health (www.nih.gov) grant

584 (AI043288) to JCC. National Science Foundation (www.nsf.gov) grant (1330562) to 585 JCC.

\section{Author Contributions}

X.Z. and J.C.C conceived and designed research. X.Z. and A.A. performed experiments. X.Z., N.F. and J.B. analyzed and plotted data. J.C.C. and X.Z. wrote the manuscript with contribution of all authors.

\section{Reference:}

591 1. Agius, C., et al., RNA silencing and antiviral defense in plants. Methods Mol Biol,

\section{894: p. 17-38.}

2. Bologna, N.G. and O. Voinnet, The diversity, biogenesis, and activities of endogenous silencing small RNAs in Arabidopsis. Annu Rev Plant Biol, 2014. 65: p. 473-503.

3. Fang, X. and Y. Qi, RNAi in Plants: An Argonaute-Centered View. Plant Cell, 2016. 28(2): p. 272-85.

4. Incarbone, M. and P. Dunoyer, RNA silencing and its suppression: novel insights from in planta analyses. Trends Plant Sci, 2013. 18(7): p. 382-92.

5. Bortolamiol, D., et al., The Polerovirus $F$ box protein $P 0$ targets ARGONAUTE1 to suppress RNA silencing. Curr Biol, 2007. 17(18): p. 1615-21. 
602 6. Pazhouhandeh, M., et al., F-box-like domain in the polerovirus protein $P 0$ is

603

604

605

606

607

608

609

610

611

612

613

614

615

616

617

618

619

620

621

622

623

624

625

626

627

628

629

630

631

632

633

634

635

636

637

638

639

640

641

642

643

644

645

646

647 required for silencing suppressor function. Proc Natl Acad Sci U S A, 2006. 103(6): p. 1994-9.

7. Chiu, M.H., et al., The silencing suppressor $P 25$ of Potato virus $X$ interacts with Argonaute 1 and mediates its degradation through the proteasome pathway. Mol Plant Pathol, 2010. 11(5): p. 641-9.

8. Baumberger, N., et al., The Polerovirus silencing suppressor P0 targets ARGONAUTE proteins for degradation. Curr Biol, 2007. 17(18): p. 1609-14.

9. Zhang, X., et al., Cucumber mosaic virus-encoded $2 b$ suppressor inhibits Arabidopsis Argonaute1 cleavage activity to counter plant defense. Genes Dev, 2006. 20(23): p. 3255-68.

10. Fang, Y.Y., et al., CMV2b-AGO Interaction Is Required for the Suppression of RDR-Dependent Antiviral Silencing in Arabidopsis. Front Microbiol, 2016. 7: p. 1329.

11. Giner, A., et al., Viral protein inhibits RISC activity by argonaute binding through conserved WG/GW motifs. PLoS Pathog, 2010. 6(7): p. e1000996.

12. Kenesi, E., et al., A viral suppressor of RNA silencing inhibits ARGONAUTE 1 function by precluding target RNA binding to pre-assembled RISC. Nucleic Acids Res, 2017. 45(13): p. 7736-7750.

13. Varallyay, E., et al., Plant virus-mediated induction of miR168 is associated with repression of ARGONAUTE1 accumulation. EMBO J, 2010. 29(20): p. 3507-19.

14. Qu, F., X. Ye, and T.J. Morris, Arabidopsis DRB4, AGO1, AGO7, and RDR6 participate in a DCL4-initiated antiviral RNA silencing pathway negatively regulated by DCL1. Proc Natl Acad Sci U S A, 2008. 105(38): p. 14732-7.

15. Garcia-Ruiz, H., et al., Roles and programming of Arabidopsis ARGONAUTE proteins during Turnip mosaic virus infection. PLoS Pathog, 2015. 11(3): p. e1004755.

16. Carbonell, A. and J.C. Carrington, Antiviral roles of plant ARGONAUTES. Curr Opin Plant Biol, 2015. 27: p. 111-7.

17. Wang, X.B., et al., The 21-nucleotide, but not 22-nucleotide, viral secondary small interfering RNAs direct potent antiviral defense by two cooperative argonautes in Arabidopsis thaliana. Plant Cell, 2011. 23(4): p. 1625-38.

18. Dzianott, A., J. Sztuba-Solinska, and J.J. Bujarski, Mutations in the antiviral RNAi defense pathway modify Brome mosaic virus RNA recombinant profiles. Mol Plant Microbe Interact, 2012. 25(1): p. 97-106.

19. Kontra, L., et al., Distinct Effects of p19 RNA Silencing Suppressor on Small RNA Mediated Pathways in Plants. PLoS Pathog, 2016. 12(10): p. e1005935.

20. Harvey, J.J., et al., An antiviral defense role of AGO2 in plants. PLoS One, 2011. 6(1): p. e14639.

21. Jaubert, M., et al., ARGONAUTE2 mediates RNA-silencing antiviral defenses against Potato virus $X$ in Arabidopsis. Plant Physiol, 2011. 156(3): p. 1556-64.

22. Scholthof, H.B., et al., Identification of an ARGONAUTE for antiviral RNA silencing in Nicotiana benthamiana. Plant Physiol, 2011. 156(3): p. 1548-55.

23. Raja, P., et al., Arabidopsis double-stranded RNA binding protein DRB3 participates in methylation-mediated defense against geminiviruses. J Virol, 2014. 88(5): p. 2611-22. 
24. Raja, P., et al., Viral genome methylation as an epigenetic defense against geminiviruses. J Virol, 2008. 82(18): p. 8997-9007.

25. Cohen, Y., A. Gisel, and P.C. Zambryski, Cell-to-cell and systemic movement of recombinant green fluorescent protein-tagged turnip crinkle viruses. Virology, 2000. 273(2): p. 258-66.

26. Cao, M., et al., The capsid protein of Turnip crinkle virus overcomes two separate defense barriers to facilitate systemic movement of the virus in Arabidopsis. $\mathrm{J}$ Virol, 2010. 84(15): p. 7793-802.

27. Donze, T., et al., Turnip crinkle virus coat protein inhibits the basal immune response to virus invasion in Arabidopsis by binding to the NAC transcription factor TIP. Virology, 2014. 449: p. 207-14.

28. Chen, Y.J., et al., The capsid protein p38 of turnip crinkle virus is associated with the suppression of cucumber mosaic virus in Arabidopsis thaliana co-infected with cucumber mosaic virus and turnip crinkle virus. Virology, 2014. 462-463: p. 71-80.

29. Thomas, C.L., et al., Turnip crinkle virus coat protein mediates suppression of RNA silencing in Nicotiana benthamiana. Virology, 2003. 306(1): p. 33-41.

30. Bock, C.H., et al., Plant Disease Severity Estimated Visually, by Digital Photography and Image Analysis, and by Hyperspectral Imaging. Critical Reviews in Plant Sciences, 2010. 29(2): p. 59-107.

31. Abbasi, A. and N. Fahlgren, Naïve Bayes pixel-level plant segmentation, in 2016 IEEE Western New York Image and Signal Processing Workshop (WNYISPW). 2016. p. 1-4.

32. Gehan, M.A., et al., PlantCV v2: Image analysis software for high-throughput plant phenotyping. PeerJ, 2017. 5: p. e4088.

33. Fahlgren, N., et al., A Versatile Phenotyping System and Analytics Platform Reveals Diverse Temporal Responses to Water Availability in Setaria. Mol Plant, 2015. 8(10): p. 1520-35.

34. Meng, C., et al., Host-induced avirulence of hibiscus chlorotic ringspot virus mutants correlates with reduced gene-silencing suppression activity. J Gen Virol, 2006. 87(Pt 2): p. 451-9.

35. Martinez-Turino, S. and C. Hernandez, Inhibition of RNA silencing by the coat protein of Pelargonium flower break virus: distinctions from closely related suppressors. J Gen Virol, 2009. 90(Pt 2): p. 519-25.

36. Sass, L., P. Majer, and E. Hideg, Leaf hue measurements: a high-throughput screening of chlorophyll content. Methods Mol Biol, 2012. 918: p. 61-9.

37. Liang, Y., et al., A nondestructive method to estimate the chlorophyll content of Arabidopsis seedlings. Plant Methods, 2017. 13: p. 26.

38. Veley, K.M., et al., High-throughput profiling and analysis of plant responses over time to abiotic stress. Plant Direct, 2017(1): p. 4.

39. Gonzalez RC, W.R., Eddins SL, Digital image processing using MATLAB. 2009, New York: Gatesmark Publishing.

40. Boyes, D.C., et al., Growth stage-based phenotypic analysis of Arabidopsis: a model for high throughput functional genomics in plants. Plant Cell, 2001. 13(7): p. $1499-510$. 
41. Nguyen, H.P., et al., Methods to Study PAMP-Triggered Immunity Using Tomato and Nicotiana benthamiana. Molecular Plant-Microbe Interactions, 2010. 23(8): p. 991-999.

42. Arend, D., et al., Quantitative monitoring of Arabidopsis thaliana growth and development using high-throughput plant phenotyping. Scientific Data, 2016. 3.

43. Lloyd, S.R., et al., Methods to Study PAMP-Triggered Immunity in Brassica Species. Molecular Plant-Microbe Interactions, 2014. 27(3): p. 286-295.

44. Laflamme, B., et al., Image-Based Quantification of Plant Immunity and Disease. Molecular Plant-Microbe Interactions, 2016. 29(12): p. 919-924.

45. Pethybridge, S.J. and S.C. Nelson, Leaf Doctor: A New Portable Application for Quantifying Plant Disease Severity. Plant Disease, 2015. 99(10): p. 1310-1316.

46. Mutka, A.M. and R.S. Bart, Image-based phenotyping of plant disease symptoms. Frontiers in Plant Science, 2015. 5.

47. Singh, A., et al., Machine Learning for High-Throughput Stress Phenotyping in Plants. Trends in Plant Science, 2016. 21(2): p. 110-124.

48. Lee, U., et al., An automated, high-throughput plant phenotyping system using machine learning based plant segmentation and image analysis. Plos One, 2018. 13(4).

49. Zhang, Z.H., et al., Arabidopsis AGO3 predominantly recruits 24-nt small RNAs to regulate epigenetic silencing. Nature Plants, 2016. 2(5).

50. Schuck, J., et al., AGO/RISC-mediated antiviral RNA silencing in a plant in vitro system. Nucleic Acids Research, 2013. 41(9): p. 5090-5103.

51. Minoia, S., et al., Specific Argonautes Selectively Bind Small RNAs Derived from Potato Spindle Tuber Viroid and Attenuate Viroid Accumulation In Vivo. Journal of Virology, 2014. 88(20): p. 11933-11945.

52. Montgomery, T.A., et al., Specificity of ARGONAUTE7-miR390 interaction and dual functionality in TAS3 trans-acting siRNA formation. Cell, 2008. 133(1): $p$. 128-141.

53. Axtell, M.J., et al., A two-hit trigger for siRNA biogenesis in plants. Cell, 2006. 127(3): p. 565-577.

54. Axtell, M.J., J.A. Snyder, and D.P. Bartell, Common functions for diverse small RNAs of land plants. Plant Cell, 2007. 19(6): p. 1750-1769.

55. Jouannet, V., et al., Cytoplasmic Arabidopsis AGO7 accumulates in membraneassociated siRNA bodies and is required for ta-siRNA biogenesis. Embo Journal, 2012. 31(7): p. 1704-1713.

56. Carbonell, A., et al., Functional Analysis of Three Arabidopsis ARGONAUTES Using Slicer-Defective Mutants. Plant Cell, 2012. 24(9): p. 3613-3629.

57. Burgyan, J. and Z. Havelda, Viral suppressors of RNA silencing. Trends in Plant Science, 2011. 16(5): p. 265-272.

58. Wu, C., et al., Analyses of RNA-Seq and sRNA-Seq data reveal a complex network of anti-viral defense in TCV-infected Arabidopsis thaliana. Scientific Reports, 2016. 6.

59. Azevedo, J., et al., Argonaute quenching and global changes in Dicer homeostasis caused by a pathogen-encoded GW repeat protein. Genes \& Development, 2010. 24(9): p. 904-915. 
60. Perez-Canamas, M. and C. Hernandez, Key Importance of Small RNA Binding for the Activity of a Glycine-Tryptophan (GW) Motif-containing Viral Suppressor of RNA Silencing. Journal of Biological Chemistry, 2015. 290(5): p. 3106-3120.

61. Deleris, A., et al., Hierarchical action and inhibition of plant Dicer-like proteins in antiviral defense. Science, 2006. 313(5783): p. 68-71.

62. Boyes, D.C., et al., Growth stage-based phenotypic analysis of arabidopsis: A model for high throughput functional genomics in plants. Plant Cell, 2001. 13(7): p. 1499-1510.

63. Thain, D., T. Tannenbaum, and M. Livny, Distributed computing in practice: the Condor experience. Concurrency and Computation-Practice \& Experience, 2005. 17(2-4): p. 323-356.

64. Schneider, C.A., W.S. Rasband, and K.W. Eliceiri, NIH Image to ImageJ: 25 years of image analysis. Nature Methods, 2012. 9(7): p. 671-675.

65. Wickham, H., Reshaping data with the reshape package. Journal of Statistical Software, 2007. 21(12): p. 1-20.

66. Wickham H, F.R., Henry L, Müller K. dplyr: A Grammar of Data Manipulation. 2017; Available from: https://dplyr.tidyverse.org/.

67. Wickham, H., ggplot2: Elegant Graphics for Data Analysis. 2009: Springer-Verlag New York.

68. Wilke, C.O. ggridges: Ridgeline Plots in 'ggplots'. 2017; Available from: https://cran.r-project.org/web/packages/ggridges/vignettes/introduction.html.

69. Team, R.C. R: A Language and Environment for Statistical Computing. 2018; Available from: https://www.r-project.org/.

\section{Supplemental Data}

S1 Fig. CPB mutation in TCV coat protein does not affect its function in virion assembly. (A) TCV virions extracted from Col-O or $d c / 2 d c / 3 d c / 4$ plants inoculated with TCV or TCV CPB were stained with Ethidium Bromide (left panel) for viral genomic RNAs or Coomassie Blue (right panel) for viral coat proteins. Virions were extracted from pooled systemic non-inoculated leaves at $14 \mathrm{dpi}$. Two biological replicates were shown in the same gel. (B) TCV virions from TCV or TCV CPB inoculum with different dilutions were stained with Ethidium Bromide (left panel) for viral genomic RNAs or Coomassie Blue (right panel) for viral coat proteins. Virions extracted from Col-O inoculated rosette leaves $(\mathrm{RL})$ and non-inoculated cauline leaves $(\mathrm{CL})$ infected with TCV were loaded and stained as positive controls.

\section{S2 Fig. Temporal visualization of rosette leaves and the corresponding pseudo-color} images in ago1-27 mutant. Plants were inoculated with mock solution, TCV or TCV CPB inoculum separately. Photos were taken from 1 day before inoculation to 17 days post inoculation (dpi). In the pseudo-color images, green color referred to healthy plant pixels, while red color referred to chlorotic/necrotic plant pixels. 
S3 Fig. Temporal visualization of rosette leaves and the corresponding pseudo-color images in ago2-1 mutant. Plants were inoculated with mock solution, TCV or TCV CPB inoculum separately. Photos were taken from 1 day before inoculation to 17 days post inoculation (dpi). In the pseudo-color images, green color referred to healthy plant pixels, while red color referred to chlorotic/necrotic plant pixels.

S4 Fig. Temporal visualization of rosette leaves and the corresponding pseudo-color images in ago3-2 mutant. Plants were inoculated with mock solution, TCV or TCV CPB inoculum separately. Photos were taken from 1 day before inoculation to 17 days post inoculation (dpi). In the pseudo-color images, green color referred to healthy plant pixels, while red color referred to chlorotic/necrotic plant pixels.

\section{S5 Fig. Temporal visualization of rosette leaves and the corresponding pseudo-color} images in ago4-2 mutant. Plants were inoculated with mock solution, TCV or TCV CPB inoculum separately. Photos were taken from 1 day before inoculation to 17 days post inoculation (dpi). In the pseudo-color images, green color referred to healthy plant pixels, while red color referred to chlorotic/necrotic plant pixels.

\section{S6 Fig. Temporal visualization of rosette leaves and the corresponding pseudo-color} images in ago5-2 mutant. Plants were inoculated with mock solution, TCV or TCV CPB inoculum separately. Photos were taken from 1 day before inoculation to 17 days post inoculation (dpi). In the pseudo-color images, green color referred to healthy plant pixels, while red color referred to chlorotic/necrotic plant pixels.

\section{S7 Fig. Temporal visualization of rosette leaves and the corresponding pseudo-color} images in ago6-3 mutant. Plants were inoculated with mock solution, TCV or TCV CPB inoculum separately. Photos were taken from 1 day before inoculation to 17 days post inoculation (dpi). In the pseudo-color images, green color referred to healthy plant pixels, while red color referred to chlorotic/necrotic plant pixels.

\section{S8 Fig. Temporal visualization of rosette leaves and the corresponding pseudo-color} images in zip-1 mutant. Plants were inoculated with mock solution, TCV or TCV CPB inoculum separately. Photos were taken from 1 day before inoculation to 17 days post inoculation (dpi). In the pseudo-color images, green color referred to healthy plant pixels, while red color referred to chlorotic/necrotic plant pixels.

S9 Fig. Temporal visualization of rosette leaves and the corresponding pseudo-color images in ago8-5 mutant. Plants were inoculated with mock solution, TCV or TCV CPB inoculum separately. Photos were taken from 1 day before inoculation to 17 days post inoculation (dpi). In the pseudo-color images, green color referred to healthy plant pixels, while red color referred to chlorotic/necrotic plant pixels.

S10 Fig. Temporal visualization of rosette leaves and the corresponding pseudo-color images in ago9-5 mutant. Plants were inoculated with mock solution, TCV or TCV CPB 
824 inoculum separately. Photos were taken from 1 day before inoculation to 17 days post

825 inoculation (dpi). In the pseudo-color images, green color referred to healthy plant pixels, while

826 red color referred to chlorotic/necrotic plant pixels.

S11 Fig. Temporal visualization of rosette leaves and the corresponding pseudo-color images in ago10-5 mutant. Plants were inoculated with mock solution, TCV or TCV CPB inoculum separately. Photos were taken from 1 day before inoculation to 17 days post inoculation (dpi). In the pseudo-color images, green color referred to healthy plant pixels, while red color referred to chlorotic/necrotic plant pixels.

S12 Fig. Temporal color change in rosette leaves in ten single ago mutant plants infected by TCV. Each histogram illustrated the distribution of pixels at each hue value (degree) in a single genotype and treatment combination at different time point. The temporal change of pixel distribution could be observed from 1 day before inoculation to $17 \mathrm{dpi}$. Pixels were collected and summed from four individual images in the same genotype and treatment combination: mock (left panel), TCV (middle panel), or TCV CPB (right panel) inoculum. The color chart illustrates the relationship between the hue value (degree) with the corresponding RGB coordinates.

S1 Table. Kolmogorov-Smirnov statistical results for rosette size curve in single ago mutants.

S2 Table. Kolmogorov-Smirnov statistical results for the percentage of unhealthy tissue curve in single ago mutants.

S3 Table. Kolmogorov-Smirnov statistical results for average hue curve in single ago mutants. ago mutants.

\section{Figures}


A.

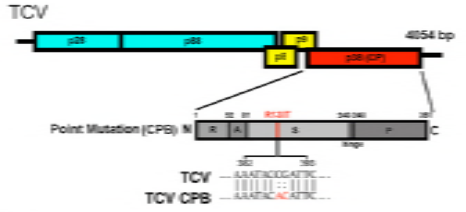

B

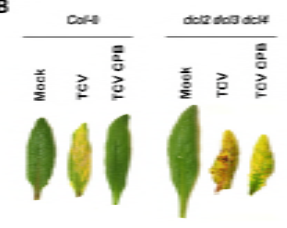

c

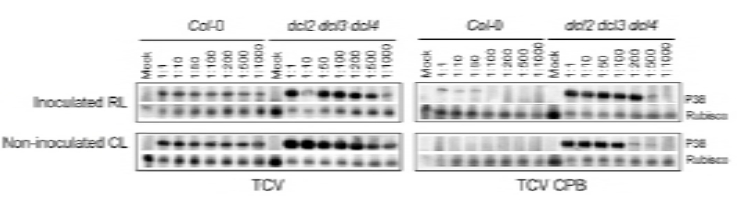

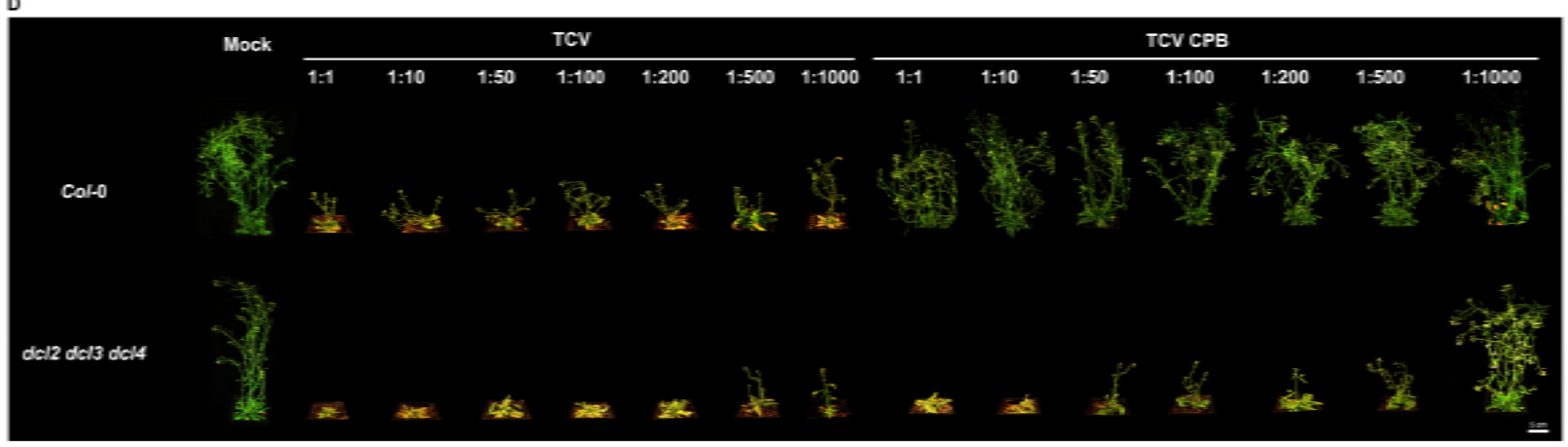

Fig 1. TCV infection-caused disease symptoms in $A$. thaliana. (A) Schematic representation of the TCV genome showing the CPB point mutation on the coat protein (P38). Top diagram shows the genomic RNA of TCV. The bottom diagram shows the coat protein (P38) region of single-amino-acid mutant TCV CPB. The single amino acid change in TCV CPB mutant is marked beneath the bottom diagram. The bottom diagram represents the full-length $\mathrm{CP}$, with the sizes and the relative positions of the five structural domains R: RNA-binding domain; A: arm domain; S: surface domain; hinge; P: protruding domain. (B) TCV infection-caused chlorosis in non-inoculated cauline leaves. Left panel: wild type control (Col-0); right panel: hyper-susceptible control ( $d c / 2 d c / 3 d c / 4)$. Photographs were taken at 14 days post inoculation (dpi). (C) Local and systemic accumulation of coat protein (P38) caused by TCV (left panel) and TCV CPB (right panel) infection was assayed by immunoblotting. P38 was detected using anti-P38 antibody. Rubisco protein was detected by anti-Rubisco antibody as a control. The virion inoculum was in different dilutions. RL: rosette leaf; CL: cauline leaf. RL samples were collected at $7 \mathrm{dpi}$; CL samples were collected at 14 dpi. (D) TCV infection-caused stunt bolt phenotype in Arabidopsis. Upper panel: wild type control (Col-0); bottom panel: hyper-susceptible control ( $d c / 2 d c / 3 d c / 4)$. The virion inoculum was in different dilutions. Photographs were taken at $14 \mathrm{dpi}$. 


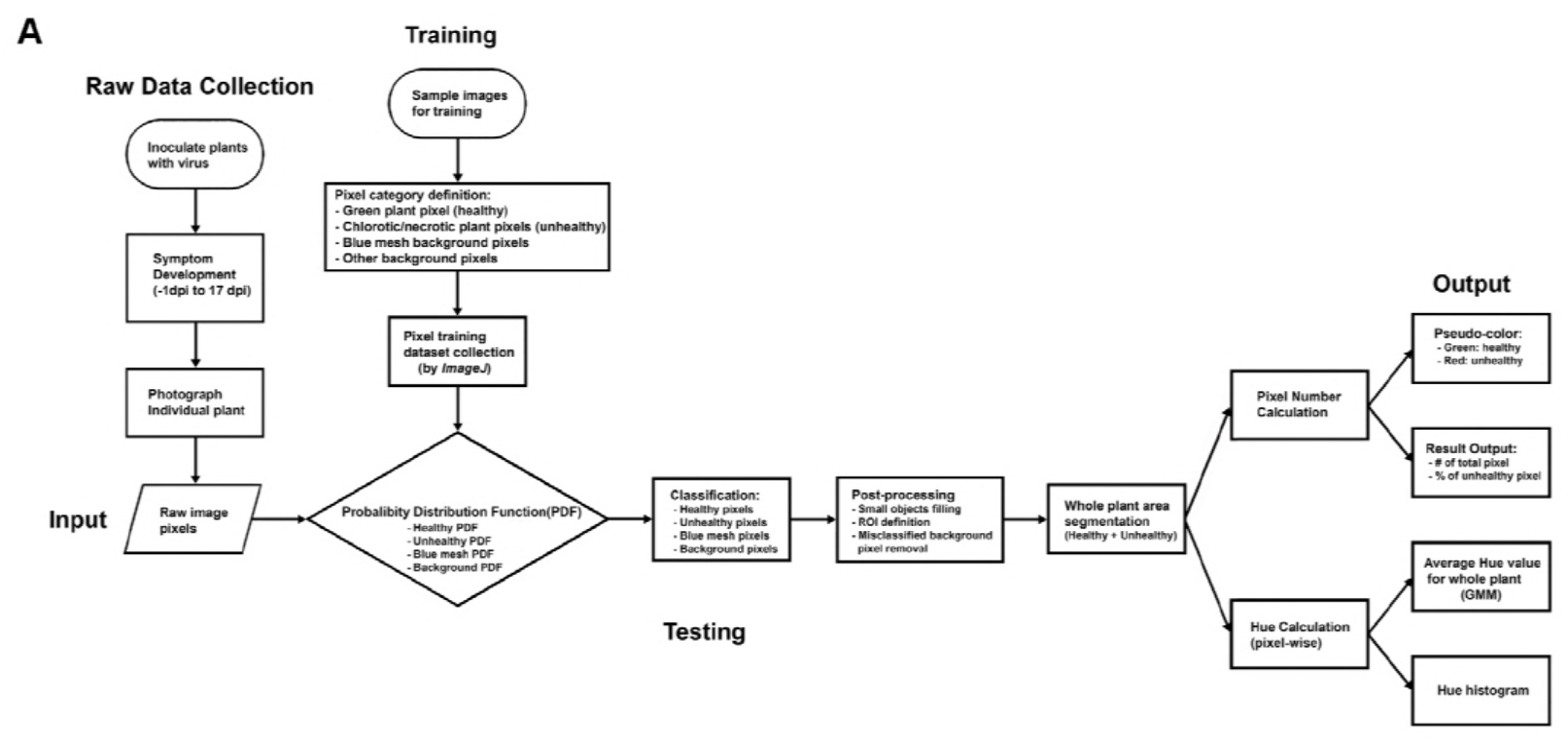

B

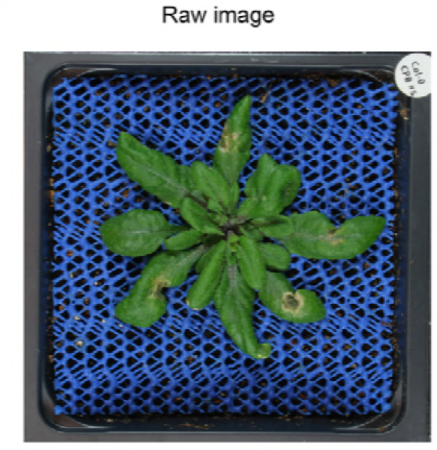

Col-0_TCV CPB_5 dpi
Segmented image

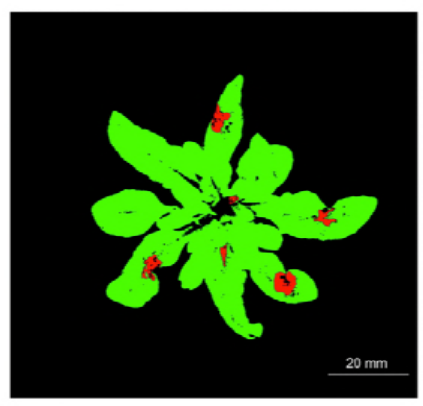

Overlaid image

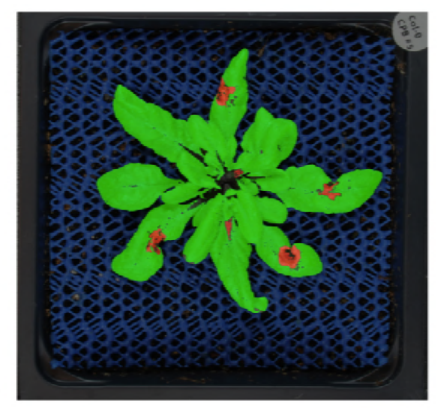

Fig 2. Image-based traits analysis workflow outline. (A) Workflow chart. Plants were inoculated with virus or mock solution. In the raw data collection section, Individual plant was photographed from 1 day before inoculation to $17 \mathrm{dpi}$. In the training session, representative sample images were chosen. Four pixel-classifiers were defined and RGB information of 1891 pixels in each class were collected to build up the training dataset. The Probability Distribution Function (PDF) for each category was calculated based on the training dataset. In the testing session, each pixel from an input image was calculated and classified into each class. After post-processing, the pipeline produced a summary of pixel number in each class and the hue value for each pixel in each raw image. Then appropriate statistics was applied to quantify and compare the output results among different groups. (B) One example of original raw plant image, segmented pseudo-color image, and overlaid image of Col-O plant inoculated with TCV CPB at 5 dpi. 

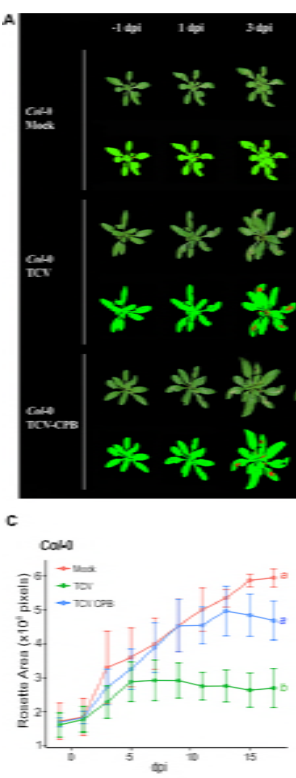

a deliz dels didelt

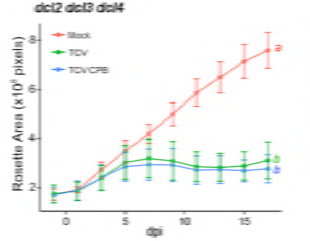

15

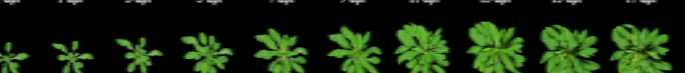

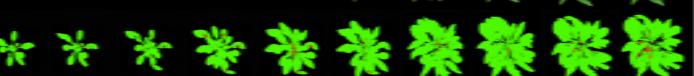

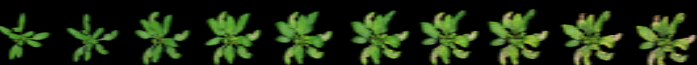

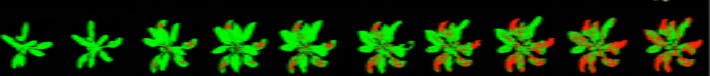

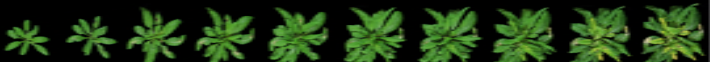

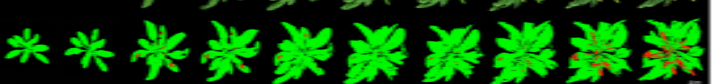

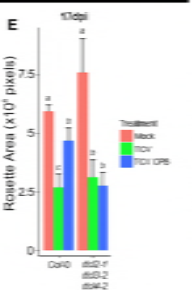

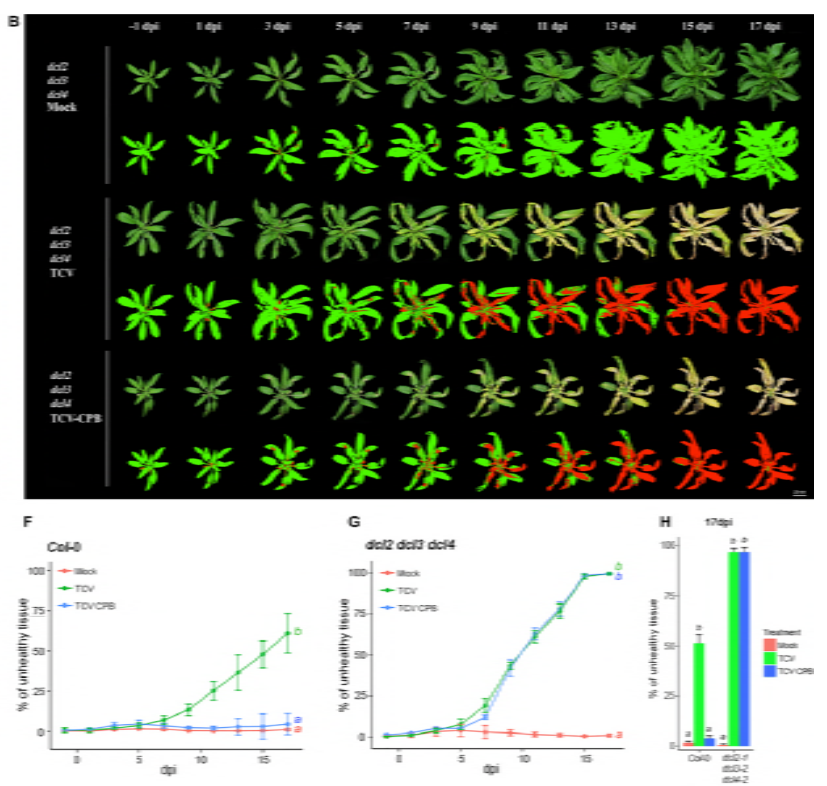

Fig 3. TCV infection-caused temporal changes in rosette size and the percentage of unhealthy tissues in control plants. (A) Temporal visualization of rosette leaves and the corresponding pseudocolor images of $\mathrm{Col}-\mathrm{O}$ and (B) $d c / 2 d c / 3 d c / 4$ plants inoculated by TCV, TCV CPB inoculum or mock solution. Photos were taken from 1 day before inoculation to $17 \mathrm{dpi}$. In the pseudo-color images, green color referred to healthy plant pixels, while red color referred to chlorotic/necrotic (unhealthy) plant pixels. (C) The growth curves show the temporal change of rosette area (total pixel number, averaged by 4 plants, $\pm \mathrm{SE}$ ) in Col-O and (D) $d c / 2 d c / 3 d c / 4$ plants from 1 day before inoculation to $17 \mathrm{dpi}$ (KolmogorovSmirnov test with $\alpha=0.05)$. ( $E$ ) The bar-plot shows the average (+ SE, $n=4)$ of rosette size at $17 \mathrm{dpi}$. Statistical analysis was calculated between treatments in each genotype. Bars with different letters are statistically different (Tukey post hoc test with $\alpha=0.05$ ). $(F)$ The curves show the percentage of unhealthy tissue change overtime (red pixels/total pixels, averaged by 4 plants, $\pm \mathrm{SE}$ ) in Col-O and (G) dc/2 dc/3 dc/4 plants from 1 day before inoculation to $17 \mathrm{dpi}$ (Kolmogorov-Smirnov test with $\alpha=0.05)$. (H) The bar-plot shows average ( $+\mathrm{SE}, \mathrm{n}=4)$ of percentage of unhealthy pixels at $17 \mathrm{dpi}$. Statistical analysis was calculated between treatments in each genotype. Bars and curves with different letters are statistically different (Tukey post hoc test with $\alpha=0.05$ ). 
A
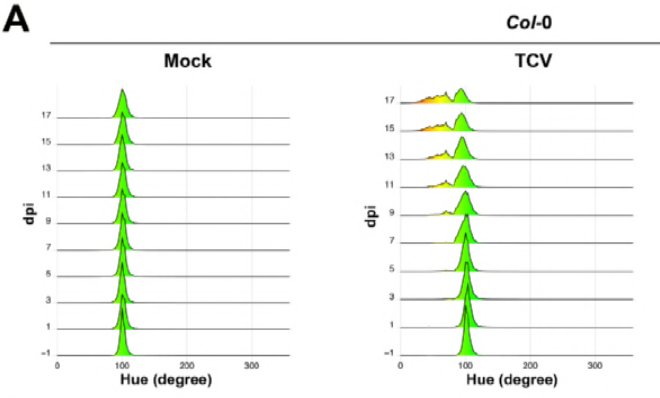

B
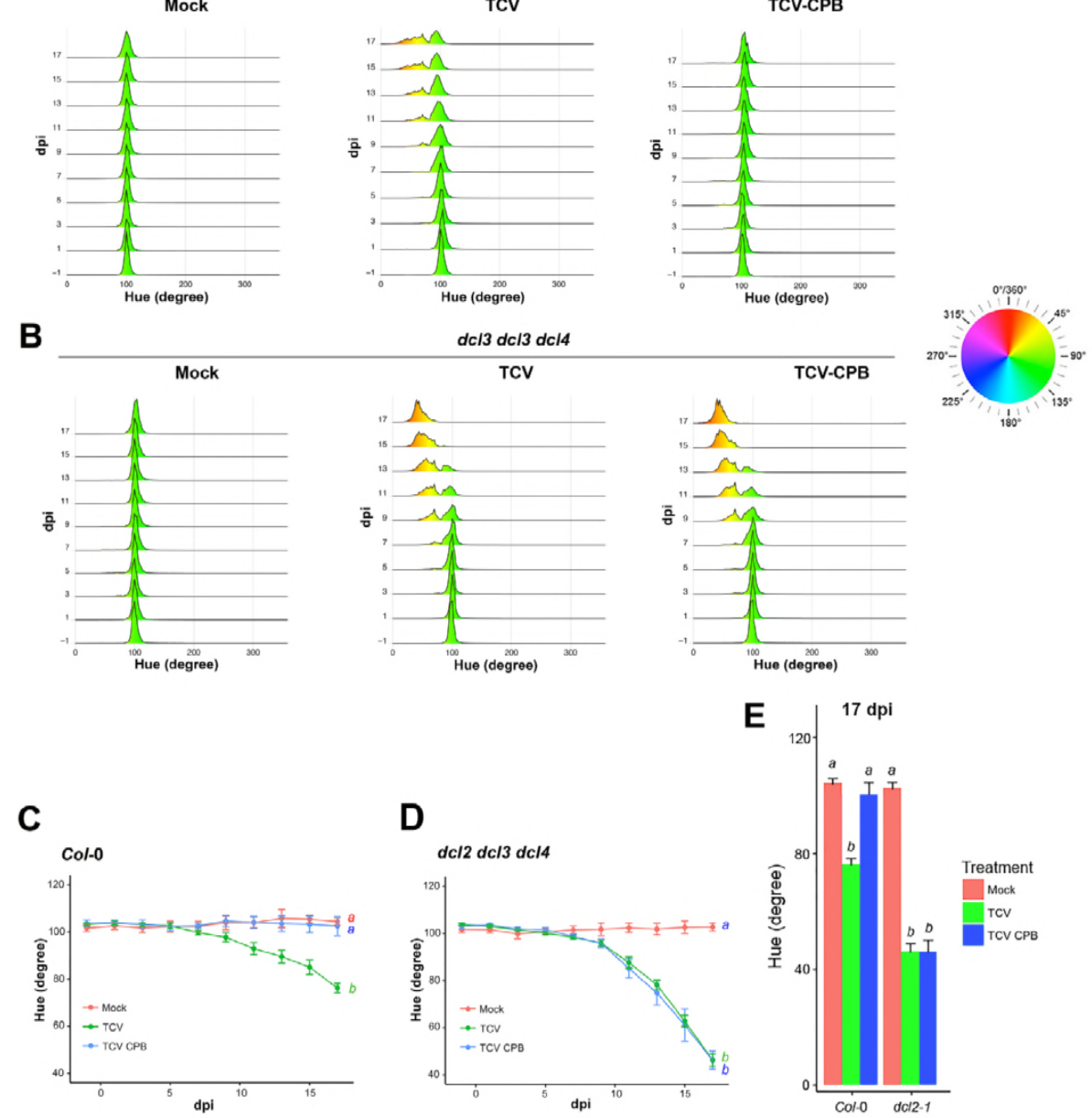

D
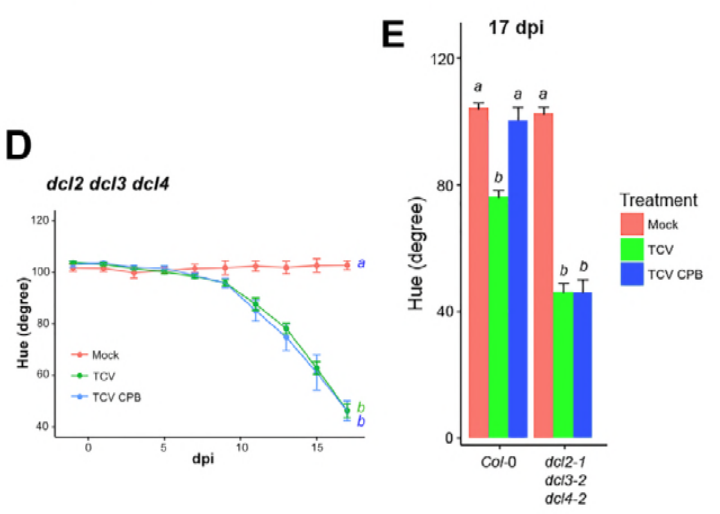

Fig 4. TCV infection-caused temporal color changes in control plants. (A) The histogram illustrates the temporal changes ( 1 day before inoculation to $17 \mathrm{dpi}$, from bottom to above) of pixel distribution at each hue value (degree) in Col- 0 and (B) $d c / 2 d c / 3 d c / 4$ plant rosette images. Pixels were collected and summed up from four individual images in the same treatment group: mock (left panel), TCV (middle panel), or TCV CPB (right panel) inoculum. The color chart illustrates the relationship between the hue value (degree) with the corresponding RGB coordinates. (C) The plots show the average hue value (degree) change over time (averaged by 4 plants, \pm SE) in Col-O and (D) dc/2 dc/3 dcl4 plants from 1 day before inoculation to $17 \mathrm{dpi}$ (Kolmogorov-Smirnov test with $\alpha=0.05)$. (E) The bar plot shows the average hue value (+ SE, $\mathrm{n}=4$ ) at $17 \mathrm{dpi}$ in $\mathrm{Col}-\mathrm{O}$ and $d c / 2 d c / 3 d c / 4$ plants. Statistical analysis was calculated between treatments in each genotype. Bars and curves with different letters are statistically different (Tukey post hoc test with $\alpha=0.05$ ). 
bioRxiv preprint doi: https://doi.org/10.1101/487322; this version posted December 4, 2018. The copyright holder for this preprint (which was not certified by peer review) is the author/funder, who has granted bioRxiv a license to display the preprint in perpetuity. It is made available under aCC-BY 4.0 International license.

A
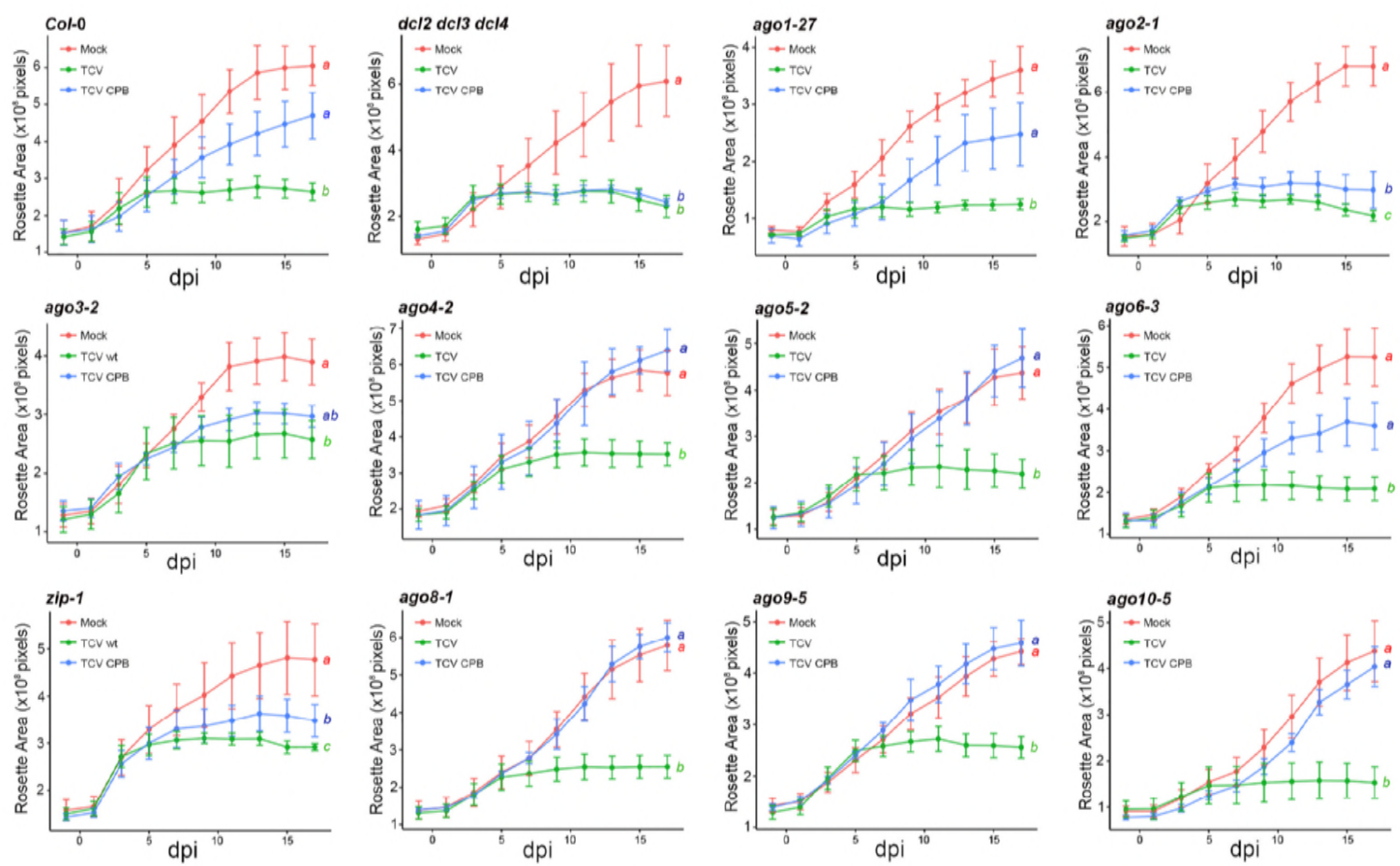

B
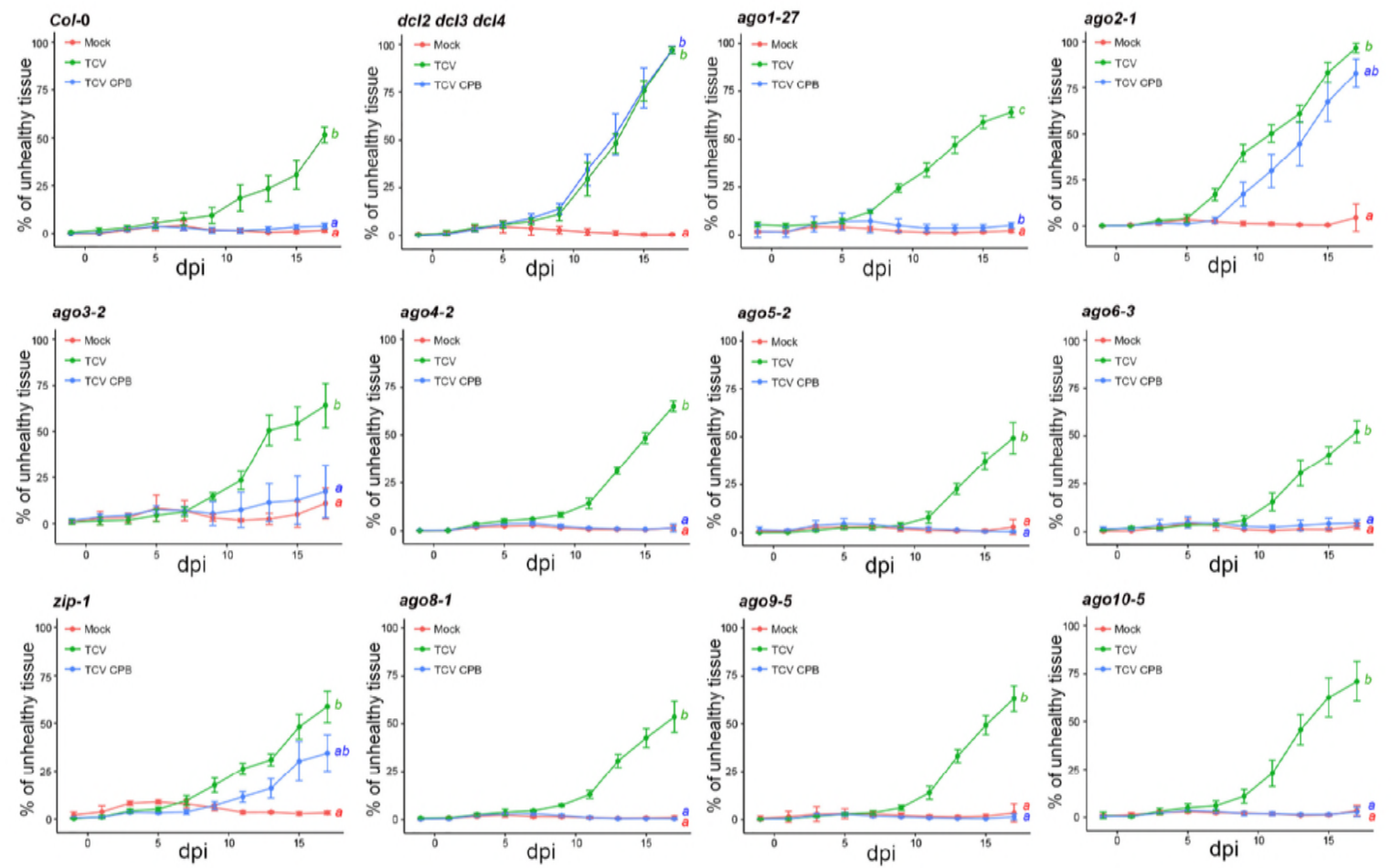
971 Fig 5. TCV infection-caused temporal changes in rosette size and the percentage of unhealthy

972 tissues in single ago mutant plants. (A) The growth curves show the temporal change of rosette area

973 (total pixel number, averaged by 4 plants, $\pm \mathrm{SE}$ ) in Col-0, dc/2 dc/3 dc/4 and ten single ago mutant plants

974 from 1 day before inoculation to $17 \mathrm{dpi}$ (Kolmogorov-Smirnov test with $\alpha=0.05$, detailed $D$ value and $p$

975 value are listed in S1 Table). (B) The curves show the percentage of unhealthy tissue change overtime

976 (red pixels/total pixels, averaged by 4 plants, \pm SE) in Col- $0, d c / 2 d c / 3 d c / 4$ and ten single ago mutant

977 plants from 1 day before inoculation to $17 \mathrm{dpi}$. (Kolmogorov-Smirnov test with $\alpha=0.05$, detailed $D$ value

978 and $p$ value are listed in S2 Table). Curves with different letters are statistically different. 
bioRxiv preprint doi: https://doi.org/10.1101/487322; this version posted December 4, 2018. The copyright holder for this preprint (which was not certified by peer review) is the author/funder, who has granted bioRxiv a license to display the preprint in perpetuity. It is made available under aCC-BY 4.0 International license.

989

990

991

992

993 


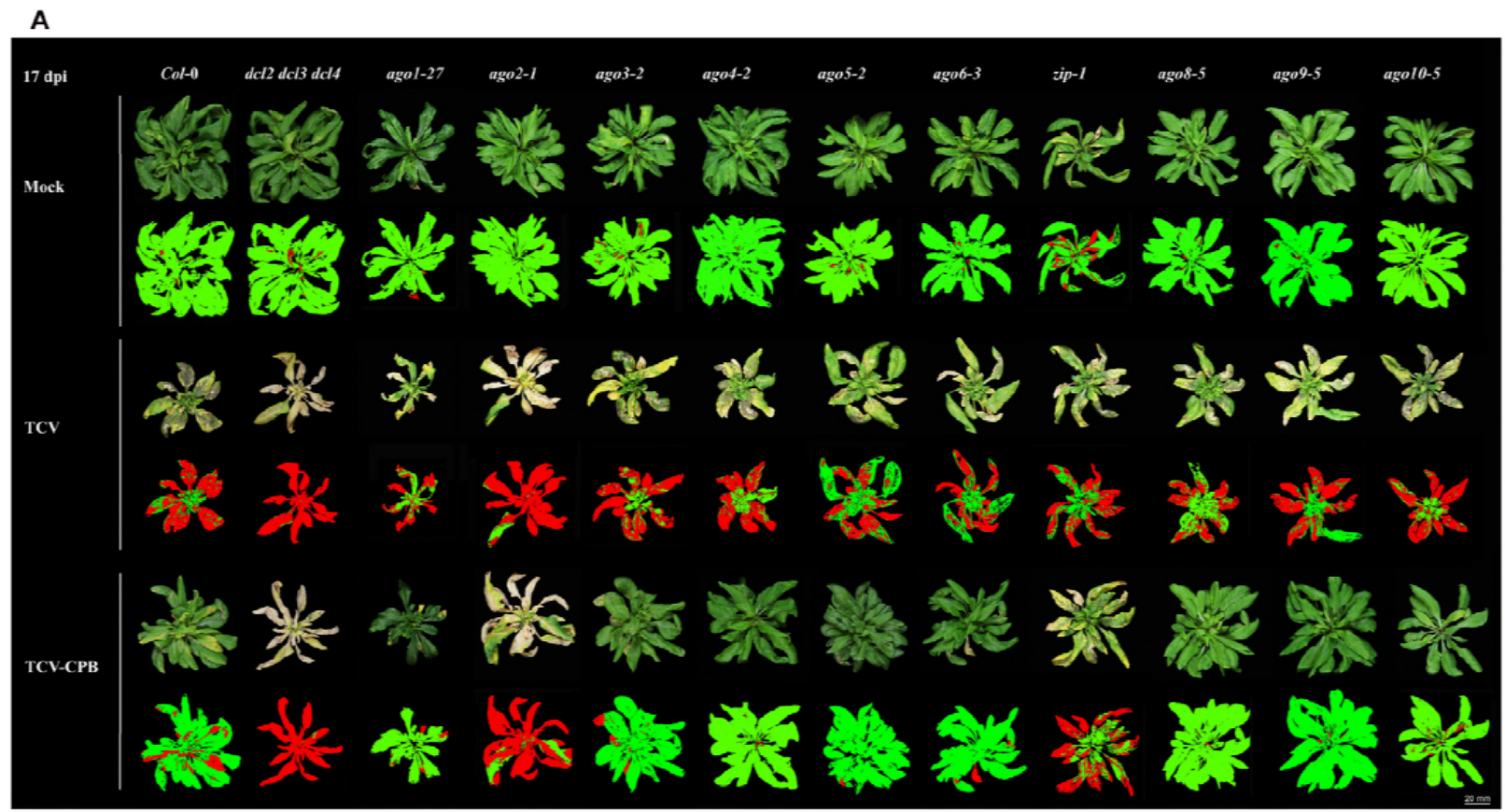

B

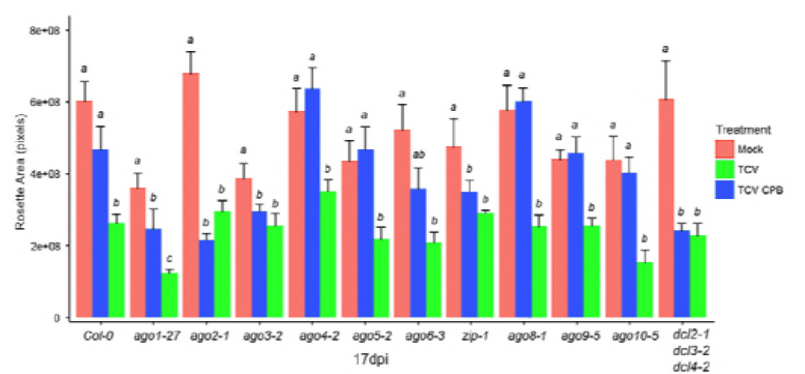

C

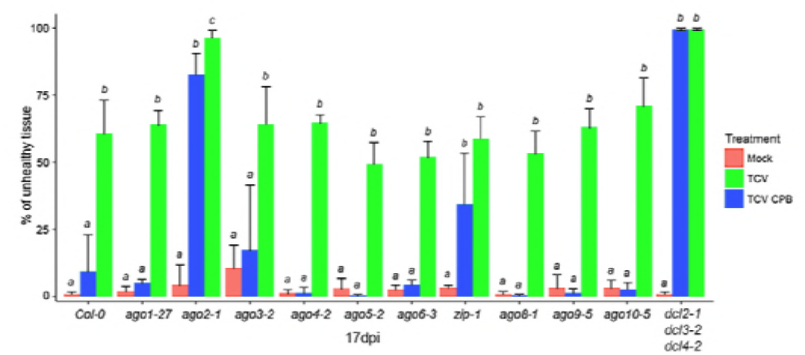

995

996

997

998

999

1000

1001

1002

1003

1004

1005

1006

1007

1008

1009

1010

1011

1012

1013

1014
Fig 6. Rosette size and the percentage of unhealthy tissue in single ago mutant plants infected with TCV at 17 dpi. (A) Representative visualization of rosette leaves and the corresponding pseudocolor images in Col-0, $d c / 2 d c / 3 d c / 4$ and ten single ago mutant plants, which were separately inoculated by mock solution, TCV or TCV CPB inoculum. Photos were taken at $17 \mathrm{dpi}$. In the pseudo-color images, green color referred to healthy plant pixels, while red color referred to chlorotic/necrotic (unhealthy) plant pixels. (B) The bar plot shows the average rosette area (total pixel number, $n=4,+\mathrm{SE}$ ) in $\mathrm{Col}-0, d c / 2 d c / 3$ $d c / 4$ and ten single ago mutant plants at $17 \mathrm{dpi}$. (C) The bar plot shows the average percentage of unhealthy tissue change overtime (red pixels/total pixels, $n=4,+\mathrm{SE}$ ) in Col- $0, d c / 2 d c / 3 d c / 4$ and single ago mutant plants at $17 \mathrm{dpi}$. Statistical analysis was calculated between treatments in each genotype. Bars with different letters are statistically different (Tukey post hoc test with $\alpha=0.05$ ). 

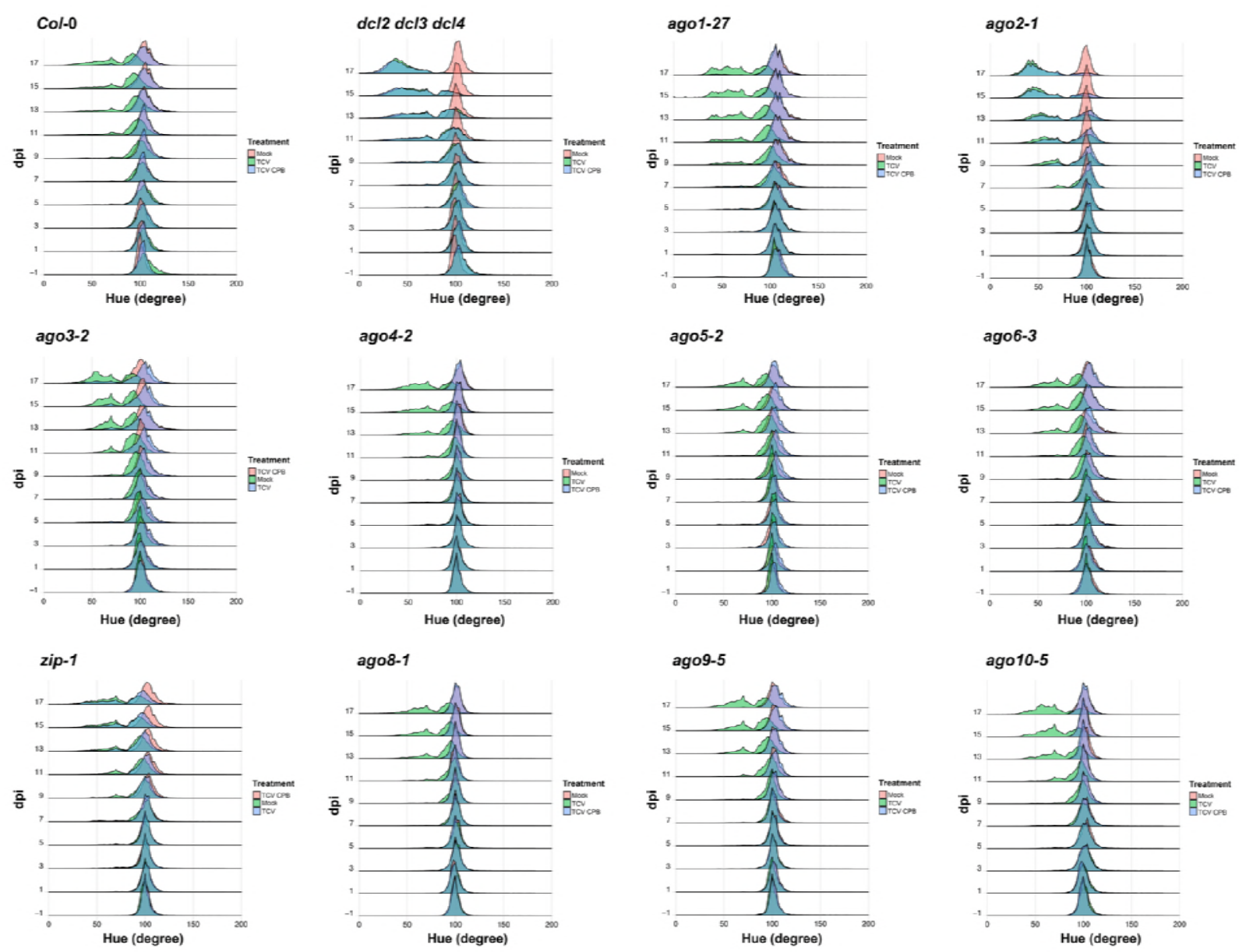

Fig 7. TCV infection-caused temporal pixel distribution changes in single ago mutant plants. Each histogram illustrates the temporal changes ( 1 day before inoculation to $17 \mathrm{dpi}$, from bottom to above) of pixel distribution at each hue value (degree) in Col-0, $d c / 2 d c / 3 d c / 4$ or the ten single ago mutant rosette images. Pixels were collected and summed up from four individual images in the same treatment group. For the separated distribution histogram in each genotype and treatment combination, refers to S12 Fig.

1023

1024

1025

1026

1027

1028

1029 

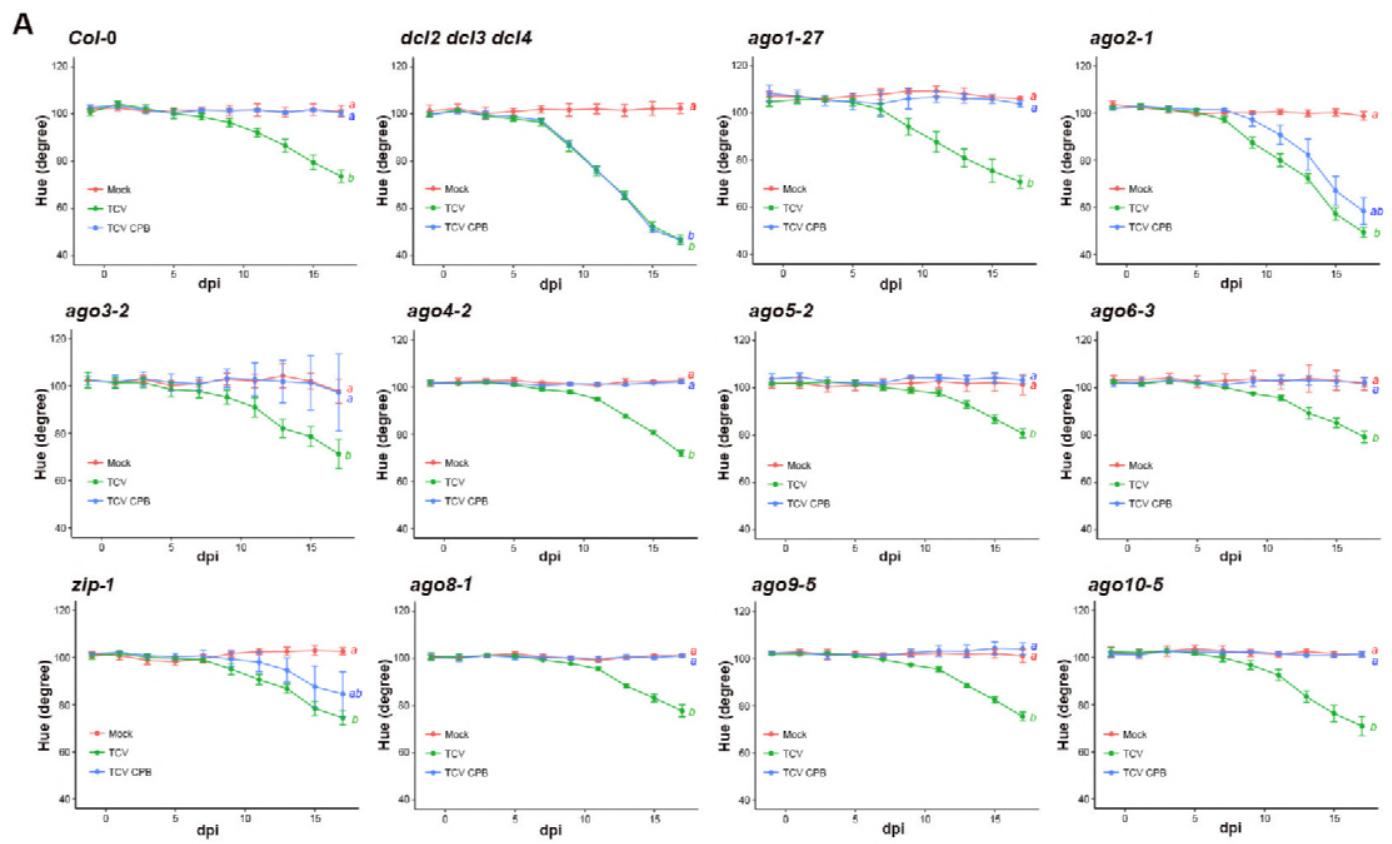

B

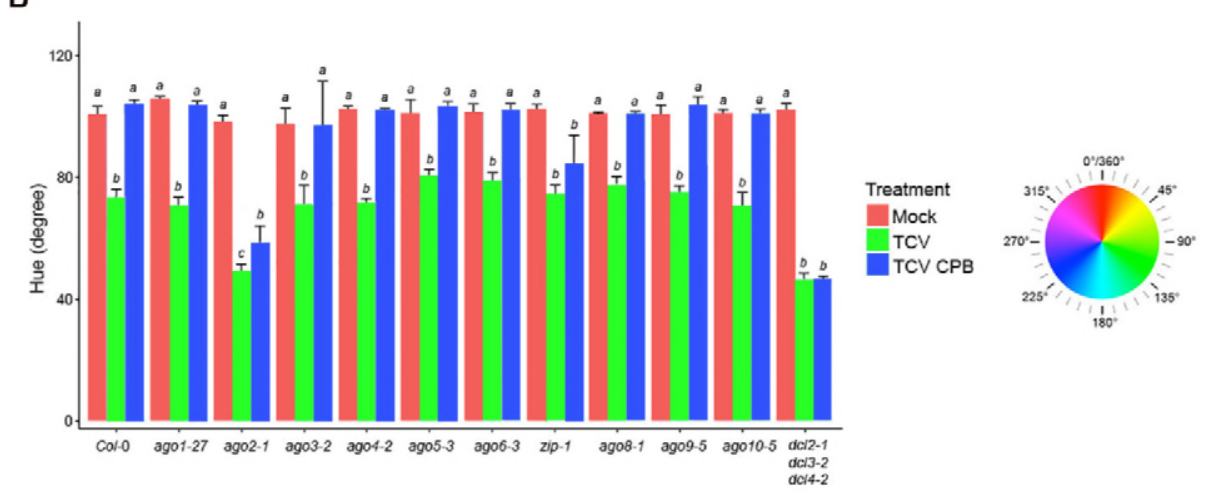

Fig 8. TCV infection-caused temporal average hue value changes in single ago mutant plants. (A) The plots show the average hue value (degree) change over time (averaged by 4 images, \pm SE) in Col-0, $d c / 2 d c / 3 d c / 4$ and single ago mutant plants from 1 day before inoculation to $17 \mathrm{dpi}$ (Kolmogorov-Smirnov test with $\alpha=0.05$, detailed $D$ value and $p$ value are listed in S3 Table). (B) The bar-plot shows the average hue value (+ SE, $n=4)$ in Col-0, $d c / 2 d c / 3 d c / 4$ and single ago mutant plants at 17 dpi. Statistical analysis was calculated between treatments in each genotype. Bars and curves with different letters are statistically different (Tukey post hoc test with $\alpha=0.05$ ). The color chart illustrates the relationship between the hue value (degree) with the corresponding RGB coordinates. 
A

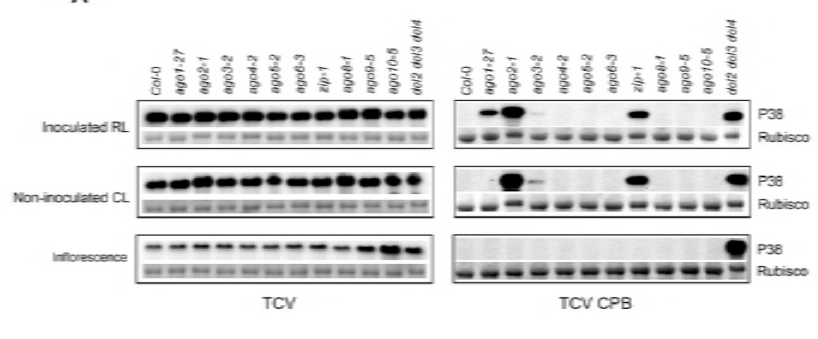

C

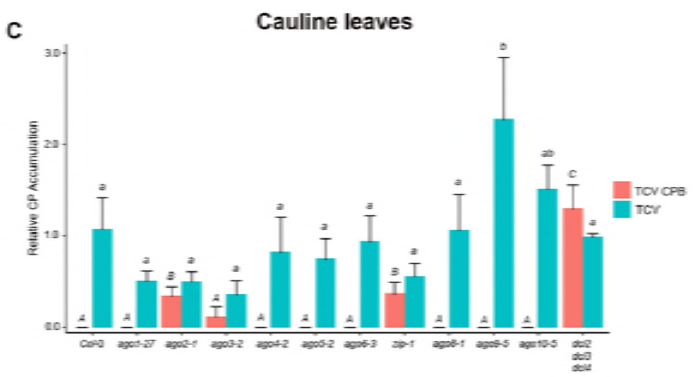

B
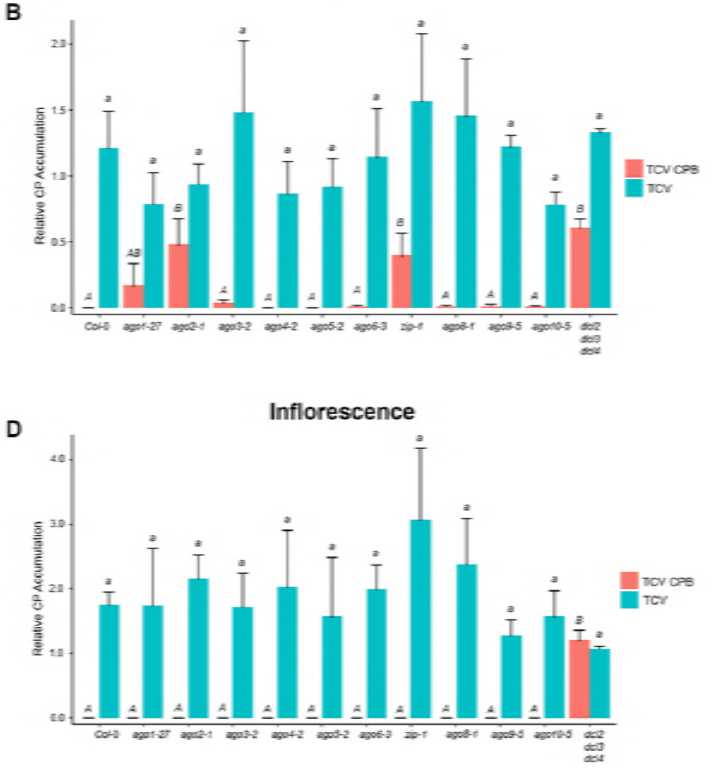

1059

1060

1061

1062

1063

1064

1065

1066

1067

1068

1069

1070

1071

1072

1073

1074

1075

1076

1077

1078

1079

1080

1081

1082

1083

1084

1085

1086

1087

1088

1089
Fig 9. Local and systemic accumulation of TCV CP in single ago mutant plants. (A) TCV viral coat protein (P38) accumulation in different tissues of Col-0, dc/2 dc/3 dcl4 and ten single ago mutant plants infected with TCV (left panel) or TCV CPB (right panel). RL: rosette leaves; CL: cauline leaves; Inflorescence. Rubisco protein: internal control. (B) Summary data: the local level of parental or CPB mutant P38 accumulation in inoculated rosette leaves of Col-O, $d c / 2 d c / 3 d c / 4$ and the ten single ago mutant plants at 7 dpi. (C) Summary data: the systemic level of parental or CPB mutant P38 accumulation in non-inoculated cauline leaves of $\mathrm{Col}-0, \mathrm{dcl} 2 \mathrm{dc} / 3 \mathrm{dc} / 4$ and the ten single ago mutant plants at $14 \mathrm{dpi}$. (D) Summary data: the systemic level of parental or CPB mutant P38 accumulation in inflorescence tissues of $\mathrm{Col}-0, \mathrm{dcl} / 2 \mathrm{dc} / 3 \mathrm{dcl} 4$ and the ten single ago mutant plants at $14 \mathrm{dpi}$. All bar plot shows average (+SE) of four biological replicates, expressed relative to $d c / 2 d c / 3 d c / 4$. Bars with different letters are statistically different (Tukey post hoc test with $\alpha=0.05$ ). 

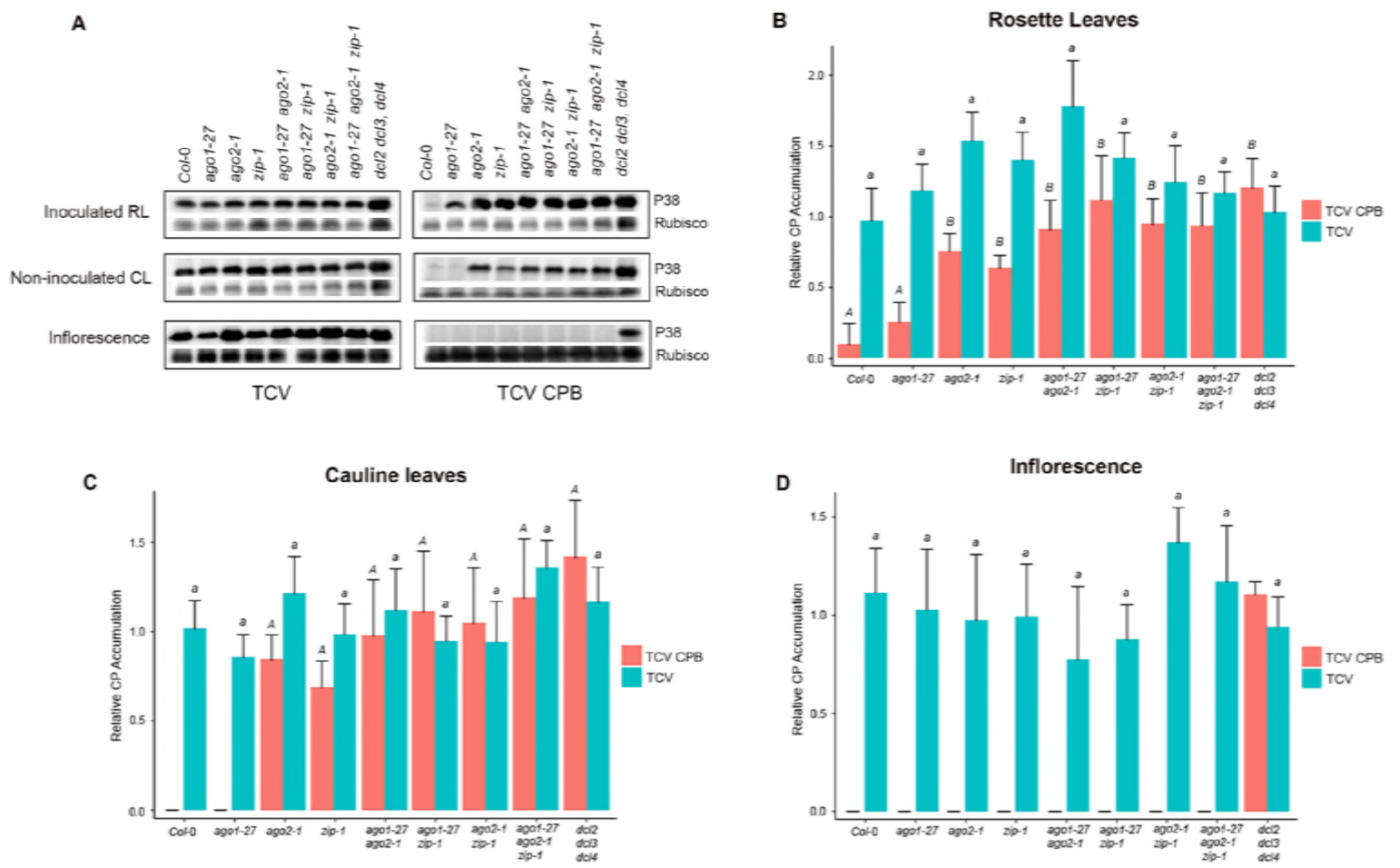

Fig 10. Local and systemic accumulation of P38 in a selected group of double and triple ago mutant plants. (A) TCV viral coat protein (P38) accumulation in different tissues of Col-0, dc/2 dc/3 dc/4 and ago1-27, ago2-1, zip-1 single, double or triple mutant plants infected with TCV (left panel) or TCV CPB (right panel). RL: rosette leaves; CL: cauline leaves; Inflorescence. Rubisco protein: internal control. (B) Summary data: the local level of parental or CPB mutant P38 accumulation in inoculated rosette leaves of Col-0, dc/2 dc/3 dc/4 and tago1-27, ago2-1, zip-1 single, double or triple mutant plants at $7 \mathrm{dpi}$. (C) Summary data: the systemic level of parental or CPB mutant P38 accumulation in non-inoculated cauline leaves of Col-0, dc/2 dc/3 dcl4 and ago1-27, ago2-1, zip-1 single, double or triple mutant plants at 14 dpi. (D) Summary data: the systemic level of parental or CPB mutant P38 accumulation in inflorescence tissues of Col-0, dcl2 dc/3 dc/4 and ago1-27, ago2-1, zip-1 single, double or triple mutant plants at $14 \mathrm{dpi}$. All bar plot shows average (+SE) of four biological replicates, expressed relative to $d c / 2$ $d c / 3 d c / 4$. Bars with different letters are statistically different (Tukey post hoc test with $\alpha=0.05$ ). 
A
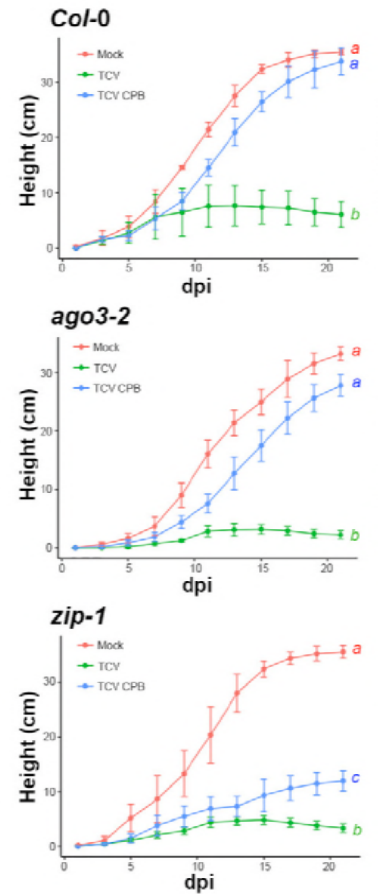

B

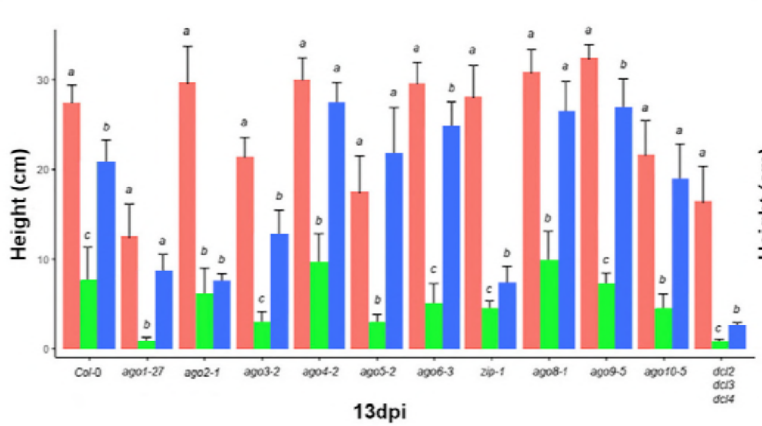

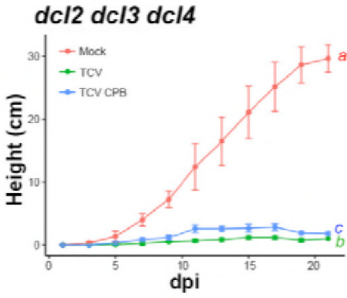
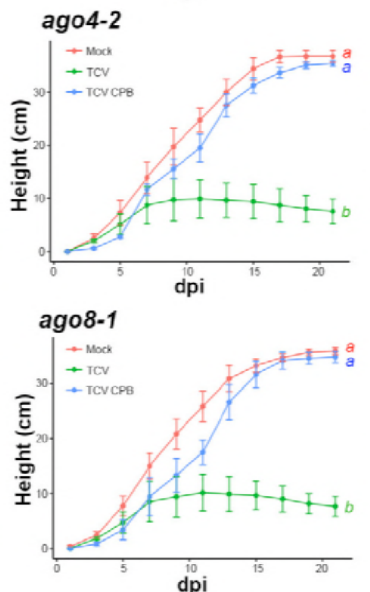

C
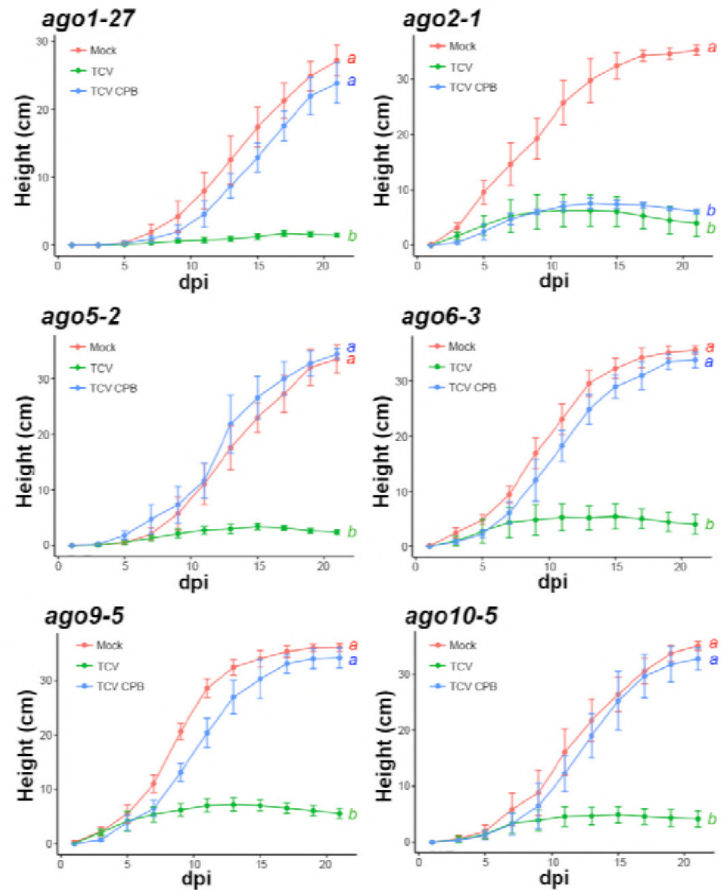

ago6-3
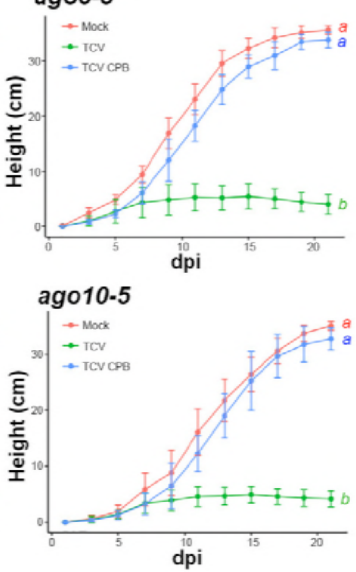

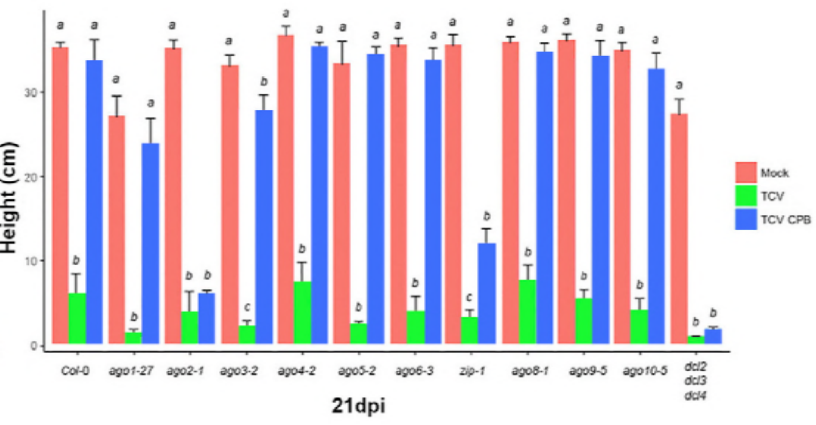

Fig 11. The effects of TCV infection on plant growth in single ago mutant plants. (A) The histograms show the plant growth curve (averaged by 6 plants, $\pm \mathrm{SE}$ ) in Col-0, $d c / 2 d c / 3 d c / 4$ and ten single ago mutant plants inoculated with mock solution, TCV or TCV CPB inoculum from one day before inoculation day to $21 \mathrm{dpi}$ (Kolmogorov-Smirnov test with $\alpha=0.05$, detailed $D$ value and $p$ value are listed in $S 4$ Table). (B) The bar plot shows the average plant height (+ SE, $\mathrm{n}=6$ ) in $\mathrm{Col}-0, d c / 2 d c / 3 d c / 4$ and ten single ago mutant plants at $13 \mathrm{dpi}$ or (C) $21 \mathrm{dpi}$. Statistical analysis was calculated between treatments in each genotype. Bars and curves with different letters are statistically different (Tukey post hoc test with 1155 $\alpha=0.05)$. 